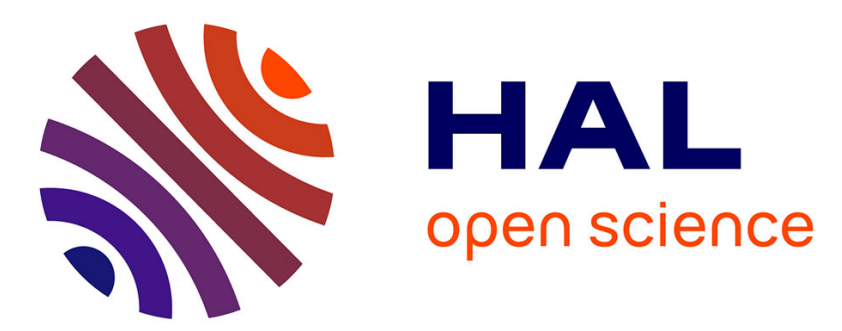

\title{
Identification of magnetic deposits in 2-D axisymmetric eddy current models via shape optimization
}

\author{
Zixian Jiang, Houssem Haddar, Armin Lechleiter, Mabrouka El-Guedri
}

\section{To cite this version:}

Zixian Jiang, Houssem Haddar, Armin Lechleiter, Mabrouka El-Guedri. Identification of magnetic deposits in 2-D axisymmetric eddy current models via shape optimization. Inverse Problems in Science and Engineering, 2015. hal-01220230

\section{HAL Id: hal-01220230 \\ https://hal.science/hal-01220230}

Submitted on 25 Oct 2015

HAL is a multi-disciplinary open access archive for the deposit and dissemination of scientific research documents, whether they are published or not. The documents may come from teaching and research institutions in France or abroad, or from public or private research centers.
L'archive ouverte pluridisciplinaire HAL, est destinée au dépôt et à la diffusion de documents scientifiques de niveau recherche, publiés ou non, émanant des établissements d'enseignement et de recherche français ou étrangers, des laboratoires publics ou privés. 


\title{
Identification of magnetic deposits in 2-D axisymmetric eddy current models via shape optimization
}

\author{
Zixian Jiang*, Houssem Haddar; Armin Lechleiter; Mabrouka El-Guedri ${ }^{\ddagger}$
}

\begin{abstract}
The non-destructive control of steam generators is an essential task for the safe and failure-free operation of nuclear power plants. Due to magnetite particles in the cooling water of the plants, a frequent source for failures are magnetic deposits in the cooling loop of steam generators. From eddy current signals measured inside a U-tube in the steam generator, we propose and analyze a regularized shape optimization algorithm to identify magnetic deposits outside the U-tube with either known or unknown physical properties. Motivated by the cylindrical geometry of the U-tubes we assume an axisymmetric problem setting, reducing Maxwell's equations to a 2-D elliptic eddy current problem. The feasibility of the proposed algorithms is illustrated via numerical examples demonstrating in particular the stability of the method under noise.

Keywords: 2-D axisymmetric eddy current model, shape optimization, boundary regularization.
\end{abstract}

\section{Introduction}

Steam generators (SGs, see Figure 1) are critical components in nuclear power plants. The reactor's core heats up water that flows through the primary loop of a SG. This primary loop consists of many thin, Ushaped tubes and serves to boil cooling water in a secondary loop on the shell side of the U-tubes. The resulting steam is then delivered to turbines generating electrical power. Due to magnetite particles contained in the cooling water, after a certain time of exploitation, conductive magnetic deposits are observed on the shell side of the Utubes. Most often, such deposits occur at the level of the support plates. They reduce the efficiency of the energy transfer between the primary and secondary loops and can harm the structure safety by clogging the water circuit between the U-tubes and the support plates.

Without disassembling the SG, the lower part of the U-tubes is inaccessible for normal inspections. Therefore, a non-destructive examination procedure, called eddy current testing (ECT), is widely used in industry to detect the presence of deposits.

In an eddy current testing procedure, one introduces a probe consisting of two coils of wire in the tube. Each of these coils is connected to a current generator producing an alternating current and to a voltmeter measuring the voltage change across the coil. One of the coils is excited by its current generator to create a primary electromagnetic field which in turn induces an eddy current in the conductive material nearby. This flow is named eddy current. The presence of conductive magnetic deposits distorts the eddy current flow and leads to a current change in the two coils, which is measured by the linked voltmeters in terms of impedance. This measurement is called ECT signal.

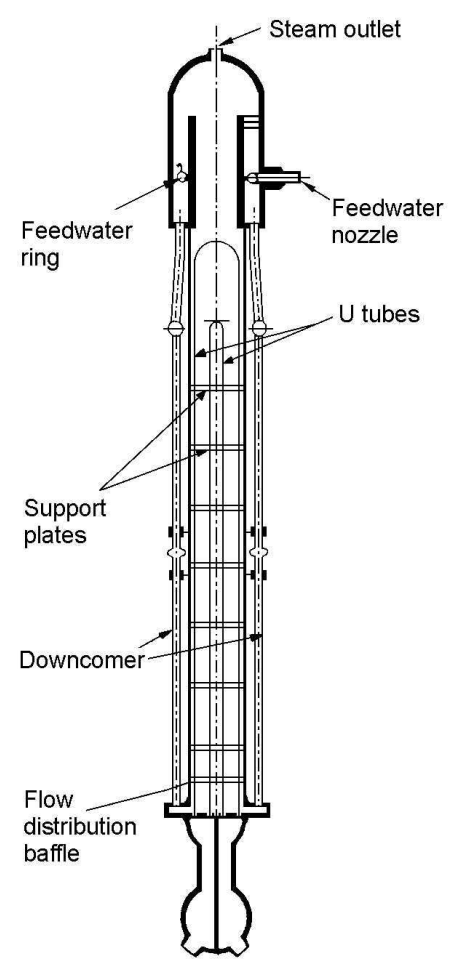

Figure 1: Steam generator

\footnotetext{
${ }^{*}$ zixian.jiang@polytechnique.edu, INRIA Saclay - CMAP Ecole Polytechnique, 91120 Palaiseau, France

${ }^{\dagger}$ houssem.haddar@inria.fr, INRIA Saclay - CMAP Ecole Polytechnique, 91120 Palaiseau, France

${ }_{\ddagger}^{\ddagger}$ lechleiter@math.uni-bremen.de, Bremen Universität, 28359 Bremen, Germany

$\S$ mabrouka.el-guedri@edf.fr, EDF R\&D, 78401 Chatou, France
} 
In the first and major part of the paper we aim to estimate the deposit shape given ECT signals supposing that the physical nature of deposit is a priori known. We shall employ for that purpose a shape optimization scheme based on evaluation of the shape derivative of the measured signal with respect to the deposit shape. We may refer to Murat and Simon [18, 19, Delfour and Zolésio [10] and Allaire [1] for a general introduction to shape optimization. The work of Pantz [21] on shape derivatives of heat equation with jumps of conductivity inspires our derivation of material derivative of eddy current equation. One can also think of other inversion strategies such as inversion methods based on topological derivatives (Guzina and Bonnet [7,12]) or the level-set approach (Santosa [23], Dorn and Lesselier [11]). Adapting these methods to the setting of our problem (for instance the backscattering measurements configuration) would be indeed of interest. From the engineering point of view, an inversion approach trying to find the linearized relationship between ECT signals and some shape parameters using finite differences is widely applied in industry $[3,5,6,8,24,26]$. This approach generally applied to detect defects characterized by limited parameters is nevertheless too restricted for general shape reconstructions.

The inversion scheme we propose combines shape derivatives with a standard gradient descent strategy to minimize a least square cost functional. In order to stabilize the gradient we regularize the descent direction by solving a Laplace-Beltrami problem on the deposit boundary. Similar regularization methods are discussed and applied in the works of Nicolas [20] and Chaulet [9]. We validate our procedure through some numerical experiments that clearly demonstrate that the ECT signals are capable to provide good estimates on the deposit shapes.

In the second part of the paper we discuss inversion schemes to reconstruct both shape and material parameters of magnetic deposits in SGs. While retrieving either the conductivity or the magnetic permeability is possible given known deposit shape, accurate simultaneous reconstruction of both parameters requires a rather good initial guess. Reconstructing the shape and one of the parameters is still rather sensitive to the initial guess. However we show that the sensitivity with respect to shape is much more robust. Reasonably accurate estimates of shape can be obtained with a small error on material parameters.

Let us briefly outline the content of the paper. Section 2 recalls the eddy current model for axisymmetric configurations and explain different impedance measurement modes and their evalution from axisymmetric eddy current models. Section 3 is then dedicated to characterizing the shape derivative of the solution and the impedance measurements with respect to the deposit shape. We also give a representation of the impedance derivative using the adjoint state technique. The shape reconstruction scheme together with numerical examples validating this scheme is given in Section 4. We then analyze in Section 5 the reconstruction of physical parameters for known geometries of the deposit. Finally, we discuss the simultaneous reconstruction of both shape and physical parameters of a deposit in Section 6 .

\section{Modeling ECT signals for axisymmetric configurations}

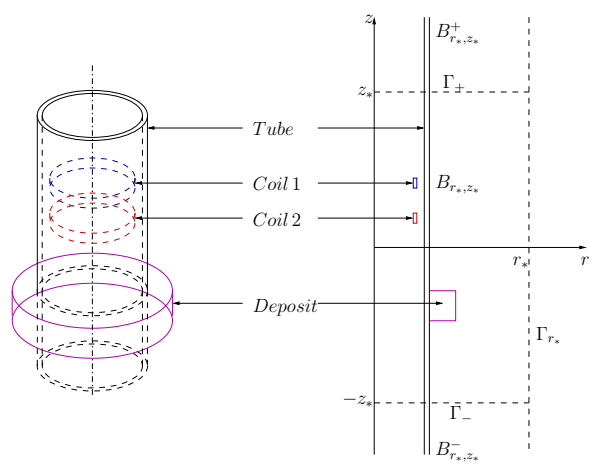

Figure 2: 3-D and 2-D representations of eddy current probe testing a tube covered by deposits.
Active coils generate an electric field $\boldsymbol{E}$ and a magnetic field $\boldsymbol{H}$ that satisfy the Maxwell's equations

$$
\left\{\begin{array}{rlrl}
\operatorname{curl} \boldsymbol{H}+(\mathrm{i} \omega \epsilon-\sigma) \boldsymbol{E} & =\boldsymbol{J} & & \text { in } \mathbb{R}^{3}, \\
\operatorname{curl} \boldsymbol{E}-\mathrm{i} \omega \mu \boldsymbol{H}=0 & & \text { in } \mathbb{R}^{3},
\end{array}\right.
$$

where $\boldsymbol{J}$ is the applied electric current density (satisfying $\operatorname{div} \boldsymbol{J}=0$ ), and $\omega, \epsilon, \mu, \sigma$ respectively denote the frequency, the electrical permittivity, the magnetic permeability and the conductivity.

In an ECT experiment, the probe consisting of two coils moves along the axis of the SG tube from vertical position $z_{\min }$ to $z_{\max }$. At each position $\zeta \in$ $\left[z_{\min }, z_{\max }\right]$, we get an impedance measurement (ECT signal) $Z_{\text {meas }}(\zeta)$. According to [4, (10a)], in the 3-D case the impedance measured in the coil $k$ when the 
electromagnetic field is induced by the coil $l$ writes

$$
\triangle Z_{k l}=\frac{1}{I^{2}} \int_{\partial \Omega_{d}^{3 \mathrm{D}}}\left(\boldsymbol{E}_{l}^{0} \times \boldsymbol{H}_{k}-\boldsymbol{E}_{k} \times \boldsymbol{H}_{l}^{0}\right) \cdot n \mathrm{~d} S,
$$

where $\Omega_{d}^{3 \mathrm{D}} \subset \mathbb{R}^{3}$ is the deposit domain, $\left(\boldsymbol{E}_{l}^{0}, \boldsymbol{H}_{l}^{0}\right)$ are the electric and magnetic fields in the deposit-free case with corresponding permeability $\mu^{0}$ and conductivity $\sigma^{0}$, while $\left(\boldsymbol{E}_{k}, \boldsymbol{H}_{k}\right)$ are the fields in the case with deposits. Using the divergence theorem and Maxwell's equations (1) one has

$$
\begin{aligned}
\triangle Z_{k l} & =\frac{1}{I^{2}} \int_{\Omega_{d}^{3 \mathrm{D}}} \operatorname{div}\left(\boldsymbol{E}_{l}^{0} \times \boldsymbol{H}_{k}-\boldsymbol{E}_{k} \times \boldsymbol{H}_{l}^{0}\right) \mathrm{d} x \\
& =\frac{1}{I^{2}} \int_{\Omega_{d}^{3 \mathrm{D}}}\left(\operatorname{curl} \boldsymbol{E}_{l}^{0} \cdot \boldsymbol{H}_{k}-\boldsymbol{E}_{l}^{0} \cdot \operatorname{curl} \boldsymbol{H}_{k}-\operatorname{curl} \boldsymbol{E}_{k} \cdot \boldsymbol{H}_{l}^{0}+\boldsymbol{E}_{k} \cdot \operatorname{curl} \boldsymbol{H}_{l}^{0}\right) \mathrm{d} x \\
& =\frac{1}{\mathrm{i} \omega I^{2}} \int_{\Omega_{d}^{3 \mathrm{D}}}\left(\left(\frac{1}{\mu}-\frac{1}{\mu^{0}}\right) \operatorname{curl} \boldsymbol{E}_{k} \cdot \operatorname{curl} \boldsymbol{E}_{l}^{0}-\left(\mathrm{i} \omega\left(\sigma-\sigma^{0}\right)+\omega^{2}\left(\epsilon-\epsilon^{0}\right)\right) \boldsymbol{E}_{k} \cdot \boldsymbol{E}_{l}^{0}\right) \mathrm{d} x .
\end{aligned}
$$

The eddy current approximation corresponds to low frequency and high conductivity regimes, that is $\omega \epsilon \ll \sigma$. Hence we get the approximation

$$
\triangle Z_{k l} \simeq \frac{1}{\mathrm{i} \omega I^{2}} \int_{\Omega_{d}^{3 \mathrm{D}}}\left(\left(\frac{1}{\mu}-\frac{1}{\mu^{0}}\right) \operatorname{curl} \boldsymbol{E}_{k} \cdot \operatorname{curl} \boldsymbol{E}_{l}^{0}-\mathrm{i} \omega\left(\sigma-\sigma^{0}\right) \boldsymbol{E}_{k} \cdot \boldsymbol{E}_{l}^{0}\right) \mathrm{d} x .
$$

In an axisymmetric setting, for a vector field $\boldsymbol{a}$ we denote by $\boldsymbol{a}_{m}=a_{r} \boldsymbol{e}_{r}+a_{z} \boldsymbol{e}_{z}$ its meridian and by $\boldsymbol{a}_{\theta}=a_{\theta} \boldsymbol{e}_{\theta}$ its azimuthal component. A vector field $\boldsymbol{a}$ is axisymmetric if $\partial_{\theta} \boldsymbol{a}$ vanishes. Then the Maxwell's equations (11) decouple into two systems, one for $\left(\boldsymbol{H}_{\theta}, \boldsymbol{E}_{m}\right)$ and the other for $\left(\boldsymbol{H}_{m}, \boldsymbol{E}_{\theta}\right)$. The solution to the first system vanishes if $\boldsymbol{J}$ is axisymmetric. Substituting $\boldsymbol{H}_{m}$ in the second system yields the second-order equation for $\boldsymbol{E}_{\theta}=E_{\theta} \boldsymbol{e}_{\theta}$,

$$
\frac{\partial}{\partial r}\left(\frac{1}{\mu r} \frac{\partial}{\partial r}\left(r E_{\theta}\right)\right)+\frac{\partial}{\partial z}\left(\frac{1}{\mu} \frac{\partial E_{\theta}}{\partial z}\right)+\omega^{2}(\epsilon+i \sigma / \omega) E_{\theta}=-i \omega J_{\theta} \quad \text { in } \mathbb{R}_{+}^{2},
$$

with $\mathbb{R}_{+}^{2}:=\{(r, z): r>0, z \in \mathbb{R}\}$. Under the eddy current approximation $(\omega \epsilon \ll \sigma)$ one has

$$
\frac{\partial}{\partial r}\left(\frac{1}{\mu r} \frac{\partial}{\partial r}\left(r E_{\theta}\right)\right)+\frac{\partial}{\partial z}\left(\frac{1}{\mu} \frac{\partial E_{\theta}}{\partial z}\right)+\mathrm{i} \omega \sigma E_{\theta}=-\mathrm{i} \omega J_{\theta} \quad \text { in } \mathbb{R}_{+}^{2},
$$

with a Dirichlet boundary condition at $r=0$ due to symmetry: $\left.E_{\theta}\right|_{r=0}=0$, and a decay condition $E_{\theta} \rightarrow 0$ as $r^{2}+z^{2} \rightarrow \infty$ at infinity. We then obtain

$$
\begin{aligned}
\triangle Z_{k l} & =\frac{2 \pi}{\mathrm{i} \omega I^{2}} \int_{\Omega_{d}}\left(\left(\frac{1}{\mu}-\frac{1}{\mu^{0}}\right) \frac{1}{r} \nabla\left(r E_{\theta, k}\right) \cdot \nabla\left(r E_{\theta, l}^{0}\right)-\mathrm{i} \omega\left(\sigma-\sigma^{0}\right) E_{\theta, k} E_{\theta, l}^{0} r\right) \mathrm{d} r \mathrm{~d} z \\
& =\frac{2 \pi}{\mathrm{i} \omega I^{2}} \int_{\Omega_{d}}\left(\left(\frac{1}{\mu}-\frac{1}{\mu^{0}}\right) \frac{\nabla w_{k} \cdot \nabla w_{l}^{0}}{r}-\mathrm{i} \omega\left(\sigma-\sigma^{0}\right) \frac{w_{k} w_{l}^{0}}{r}\right) \mathrm{d} r \mathrm{~d} z,
\end{aligned}
$$

where we have set

$$
w_{j}:=r E_{\theta, j}, w_{j}^{0}:=r E_{\theta, j}^{0}, j=1,2 .
$$

We shall assume that $\mu$ and $\sigma$ are in $L^{\infty}\left(\mathbb{R}_{+}^{2}\right)$ such that $\mu \geq \mu_{v}>0$ on $\mathbb{R}_{+}^{2}$ and that $\sigma \geq 0$ and $\sigma=0$ for $r \geq r_{0}$ sufficiently large. Then problem (3) has a unique solution $E_{\theta} \in H\left(\mathbb{R}_{+}^{2}\right)$ if one assumes for instance that $J_{\theta} \in L^{2}\left(\mathbb{R}_{+}^{2}\right)$ with compact support where we used the notation for any $\Omega \subset \mathbb{R}_{+}^{2}$

$$
H(\Omega):=\left\{v: r^{1 / 2}\left(1+r^{2}\right)^{-\lambda / 2} v \in L^{2}(\Omega), r^{-1 / 2} \nabla(r v) \in L^{2}(\Omega)\right\}
$$

where $\lambda$ can be any real $>1$ and where $\nabla:=\left(\partial_{r}, \partial_{z}\right)^{t}$ (see [13, Proposition 2.2] for detailed proof of the well-posedness of problem (3), or more precisely, of its equivalent variational formulation). In the 
following it will be more convenient to work with $w:=r E_{\theta} \in \widetilde{H}(\Omega):=\{v: r v \in H(\Omega)\}$. This field satisfies the variational formulation

$$
a(w, \varphi):=\int_{\Omega}\left(\frac{1}{\mu r} \nabla w \cdot \nabla \bar{\varphi}-\frac{\mathrm{i} \omega \sigma}{r} w \bar{\varphi}\right) \mathrm{d} r \mathrm{~d} z=\int_{\Omega} \mathrm{i} \omega J \bar{\varphi} \mathrm{d} r \mathrm{~d} z \quad \forall \varphi \in \widetilde{H}(\Omega)=\{v: r v \in H(\Omega)\}
$$

with $\Omega=\mathbb{R}_{+}^{2}$ and $J=J_{\theta}$. The solution to (5) satisfies (in the weak sense)

$$
-\operatorname{div}\left(\frac{\nabla w}{\mu r}\right)-\mathrm{i} \omega \sigma \frac{w}{r}=\mathrm{i} \omega J \quad \text { in } \Omega .
$$

Let us already indicate that for numerical purposes, the computational domain will be truncated in radial direction at $r=r_{*}$ where $r_{*}$ is sufficiently large and impose a Neumann boundary condition on $r=r_{*}$ (see Figure 2). Then the solution for the truncated problem would satisfy (5) with $\Omega=B_{r_{*}}:=$ $\left\{(r, z) \in \mathbb{R}^{2}: 0 \leq r \leq r_{*}\right\}$. This is why we shall use in the sequel the variational formulation (5) with the generic notation for the variational space $\widetilde{H}(\Omega)$ with $\Omega$ denoting $\mathbb{R}_{+}^{2}$ or $B_{r_{*}}$. We also recall that the variational formulation with $\Omega=B_{r_{*}}$ can be equivalently reduced to a variational formulation posed on $B_{r_{*}, z_{*}}=\left\{(r, z) \in \mathbb{R}^{2}: 0 \leq r \leq r_{*},|z|<z_{*}\right\}$ by introducing appropriate Dirichlet-to-Neumann operators on $z= \pm z_{*}$. This would be convenient for accelerating numerical evaluation of the solution (see [13]). As a corollary of the well-posedness of the problem (3) for $E_{\theta}$ we can state:

Corollary 2.1. Assume that $\mu$ and $\sigma$ are in $L^{\infty}\left(\mathbb{R}_{+}^{2}\right)$ such that $\mu \geq \mu_{v}>0$ on $\mathbb{R}_{+}^{2}$ and that $\sigma \geq 0$ and $\sigma=0$ for $r \geq r_{0}$ sufficiently large. If the source $J \in L^{2}(\Omega)$ with compact support, then the variational formulation (5) has a unique solution $w$ in $\widetilde{H}(\Omega)$.

Let us finally note that in practice, the impedances are measured either in the absolute mode, denoted by $Z_{F A}$, or in the differential mode, denoted by $Z_{F 3}$. From [22], we have

$$
\left\{\begin{array}{rr}
Z_{F A}=\frac{\mathrm{i}}{2}\left(\triangle Z_{11}+\triangle Z_{21}\right) & \text { absolute mode, } \\
Z_{F 3}=\frac{\mathrm{i}}{2}\left(\triangle Z_{11}-\triangle Z_{22}\right) & \text { differential mode }
\end{array}\right.
$$

Notation: In the 2-D axisymmetric configuration in the Orz plan (Figure 3), the tube is represented by $\Omega_{t}:=\left\{(r, z) \in \Omega: r_{t_{1}}<r<r_{t_{2}}\right\}$ with $0<r_{t_{1}}<r_{t_{2}}$ the inner and outer radius of the tube wall. We denote by $\Omega_{s}$ the domain inside the tube $\left(r<r_{t_{1}}\right)$ which contains the support of the source: $\operatorname{supp} J \subset \Omega_{s}$. The deposit is at the shell side of the tube, that is $\Omega_{d} \subset\left\{(r, z) \in \Omega: r>r_{t_{2}}\right\}$. We denote by $\Omega_{v}$ the vacuum domain outside the tube $\Omega_{v}:=\left\{(r, z) \in \Omega: r>r_{t_{2}}\right\} \backslash \Omega_{d}$. Then we have $\Omega=\cup_{i \in \Lambda} \Omega_{i}$ where $\Lambda=\{s, t, d, v\}$ is a set of index designating the above subdomains of $\Omega$. We will also use the notation $\Omega_{d}^{\complement}$ for the complement set of $\Omega_{d}$ in $\Omega\left(\Omega_{d}^{\complement}=\Omega \backslash \Omega_{d}=\Omega_{s} \cup \Omega_{t} \cup \Omega_{v}\right)$.

Remark 2.2. If we assume that $\mu$ and $\sigma$ are piecewise constant in each subdomain $\Omega_{i}, i \in \Lambda=\{s, t, d, v\}$, then the solution $w$ to problem (5) belongs to $H^{2}(\mathcal{Q})$ for any regular open subset $\mathcal{Q}$ of $\Omega_{d}$ or $\Omega_{v}$. (Such piecewise constant material parameters indeed meet the real setting of ECT in steam generators.) This regularity property is due to [16, Chap.2Th.3.2], as $\overline{\Omega_{d} \cup \Omega_{v}}=\left\{(r, z) \in \Omega: r \geq r_{t_{2}}\right\}$ is bounded away from $\{r=0\}$ and $J \in L^{2}(\Omega)$.

If we assume in addition that the boundaries $\partial \Omega_{i}(i \in\{d, v\})$ have $C^{1,1}$-regularity, then $\left.w\right|_{\Omega_{i}}(i \in\{d, v\})$ has $H^{2}$-regularity till $\partial \Omega_{i}$, in par-

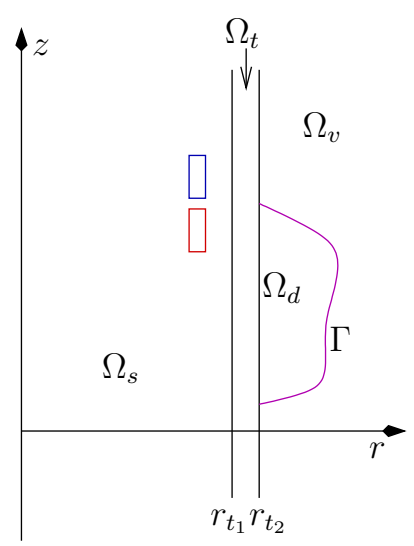

Figure 3: Sketch of an EC$\mathrm{T}$ problem of steam generator deposit in 2-D axisymmetric setting. ticular till their interface $\Gamma:=\overline{\partial \Omega_{d}} \cap \overline{\partial \Omega_{v}}$ (see [17, Theorem 4.20]). Hence the traces of $\left.\nabla w\right|_{\Omega_{i}}(i \in\{d, v\})$ on $\Gamma$ are well defined and belong to $H^{1 / 2}(\Gamma)^{2}$. 


\section{Shape derivative of the impedance measurements}

The gradient descent for shape reconstruction with a least square cost functional in the next section is based on the knowledge of the shape derivative of impedance measurements. Due to (4), we shall first study the derivatives of the shape-dependent function $w$, solution to the eddy current problem (5).

\subsection{Shape and material derivatives of the solution}

For $\mathcal{Q}$ a regular open subset of $\Omega \subset \mathbb{R}^{2}$, we define a domain deformation as a perturbation of the identity

$$
\operatorname{Id}+\theta: \mathcal{Q} \rightarrow \mathcal{Q}_{\theta}=(\operatorname{Id}+\theta) \mathcal{Q}, \quad \text { with } \theta \in W^{1, \infty}\left(\mathbb{R}^{2}, \mathbb{R}^{2}\right) \quad \text { and }\|\theta\|_{W^{1, \infty}\left(\mathbb{R}^{2}, \mathbb{R}^{2}\right)}<1 .
$$

Then Id $+\theta$ is a diffeomorphism in $\mathbb{R}^{2}$ (see [1, Lemme 6.13]). In our problem, an admissible deformation should keep the domains $\Omega_{t}$ and $\Omega_{s}$ invariant, i.e., $\operatorname{supp} \theta \cap \Omega_{s}=\operatorname{supp} \theta \cap \Omega_{t}=\emptyset$. Indeed we are mainly interested in perturbation fields $\theta$ with support located in vicinity of the interface $\Gamma=\overline{\partial \Omega_{d}} \cap \overline{\partial \Omega_{v}}$ between the deposit and the vacuum region outside the tube. We denote by $[\cdot]$ the jump operator across $\Gamma$, i.e. for any $f(x)(x=(r, z))$ defined in a vicinity of $\Gamma$ and any $x_{0}=\left(r_{0}, z_{0}\right) \in \Gamma$

$$
\begin{aligned}
& {[f]\left(x_{0}\right):=f_{+}\left(x_{0}\right)-f_{-}\left(x_{0}\right),} \\
& \text { with } f_{+}\left(x_{0}\right)=\lim _{\Omega_{v} \ni x \rightarrow x_{0}} f(x) \text { and } f_{-}\left(x_{0}\right)=\lim _{\Omega_{d} \ni x \rightarrow x_{0}} f(x) .
\end{aligned}
$$

Following [1, Section 6.3.3] we give the following definitions.

Definition 3.1. Let $v=v(\mathcal{Q})$ be a shape-dependent function that belongs to some Banach space $B$ (that may depend on $\mathcal{Q})$. If $\tilde{v}(\theta):=v\left(\mathcal{Q}_{\theta}\right) \circ(\operatorname{Id}+\theta) \in B$, then the material (Lagrangian) derivative $V(\theta)$ of $v$ is defined as a linear functional with respect to $\theta$ with values in $B$ such that

$$
\tilde{v}(\theta)=\tilde{v}(0)+V(\theta)+o(\theta) \quad \text { in } \mathcal{Q},
$$

where $\lim _{\theta \rightarrow 0} \frac{\|o(\theta)\|_{B}}{\|\theta\|_{1, \infty}}=0$. The shape (Eulerian) derivative $v^{\prime}(\theta)$ of $v$ is defined by

$$
v^{\prime}(\theta)=V(\theta)-\theta \cdot \nabla v(\mathcal{Q})
$$

In the sequel we shall adopt the generic notation $o(\theta)$ to design a function such that $\|o(\theta)\| /\|\theta\|_{1, \infty} \rightarrow 0$ as $\theta \rightarrow 0$ where the norm $\|\cdot\|$ for $o(\theta)$ should be clear from the context.

Remark 3.2. Using the chain rule it is readily seen from Definition 3.1 that formally

$$
v\left(\mathcal{Q}_{\theta}\right)=v(\mathcal{Q})+v^{\prime}(\theta)+o(\theta) \quad \text { in } \omega \subset \mathcal{Q} \cap \mathcal{Q}_{\theta}
$$

Proposition 3.3. Under the same assumptions as in Corollary 2.1, for any admissible shape perturbation $\theta \in W^{1, \infty}\left(\mathbb{R}^{2}, \mathbb{R}^{2}\right)$ with $\|\theta\|_{W^{1, \infty}}<1$, the solution $w(\Omega)$ of (5) has the material derivative $W(\theta)$ satisfying

$$
a(W(\theta), \phi)=L_{\theta}(\phi) \quad \forall \phi \in \widetilde{H}(\Omega),
$$

where $L_{\theta}(\phi):=\int_{\Omega}\left\{\frac{1}{\mu}\left(-\operatorname{div}(\theta / r) I+\frac{\nabla \theta+\nabla \theta^{t}}{r}\right) \nabla w \cdot \nabla \bar{\phi}+\mathrm{i} \omega \sigma \operatorname{div}(\theta / r) w \bar{\phi}+\mathrm{i} \omega \operatorname{div}(J \theta) \bar{\phi}\right\} \mathrm{d} r \mathrm{~d} z$.

Proof. We consider the change of variables $(\operatorname{Id}+\theta)^{-1}: \Omega_{\theta} \ni y \mapsto x \in \Omega$, and in particular the fact that

$$
(\nabla v) \circ(\operatorname{Id}+\theta)=(I+\nabla \theta)^{-t} \nabla(v \circ(\operatorname{Id}+\theta))=(I+\nabla \theta)^{-t} \nabla \tilde{v}(\theta) \quad \forall v \in \widetilde{H}\left(\Omega_{\theta}\right),
$$

where $\nabla \theta$ is the Jacobian matrix of $\theta$. Since $w\left(\Omega_{\theta}\right)$ satisfies the variational problem (5) in $\Omega_{\theta}$, one gets after the change of variable,

$$
\begin{aligned}
& \qquad \int_{\Omega}\left(\frac{1}{r}+\nabla \frac{1}{r} \cdot \theta+o(\theta)\right)\left(\frac{1}{\mu} A(\theta) \nabla \tilde{w}(\theta) \cdot \bar{\phi}-\mathrm{i} \omega \sigma \tilde{w}(\theta) \bar{\phi}|\operatorname{det}(I+\nabla \theta)|\right) \mathrm{d} r \mathrm{~d} z \\
& =\int_{\Omega} \mathrm{i} \omega J \circ(\operatorname{Id}+\theta) \bar{\phi}|\operatorname{det}(I+\nabla \theta)| \mathrm{d} r \mathrm{~d} z, \\
& \text { with } \quad A(\theta):=|\operatorname{det}(I+\nabla \theta)|(I+\nabla \theta)^{-1}\left((I+\nabla \theta)^{-1}\right)^{t}, \quad \phi:=\varphi \circ(\operatorname{Id}+\theta) .
\end{aligned}
$$


Expanding the above formulation with respect to $\theta$ and using the identities

$$
\operatorname{det}(I+\theta)=1+\operatorname{div} \theta+o(\theta), \quad(I+\nabla \theta)^{-1}=I-\nabla \theta+o(\theta),
$$

the terms of order zero with respect to $\theta$ give exactly the variational formulation on $\Omega$ (5), while the first order terms with respect to $\theta$ yield the formulation (9). Since the sesquilinear form $a(\cdot, \cdot)$ is continuous and coercive, the variational formulation (9) has a unique solution.

To simplify the variational formulation (9), we shall prove some preliminary technical results. For any regular open subset $\mathcal{Q} \subset \Omega$, we define a shape-dependent sesquilinear form

$$
\alpha(\mathcal{Q})(u(\mathcal{Q}), v(\mathcal{Q})):=\int_{\mathcal{Q}}\left(\frac{1}{\mu r} \nabla u \cdot \nabla \bar{v}-\frac{\mathrm{i} \omega \sigma}{r} u \bar{v}\right) \mathrm{d} r \mathrm{~d} z \quad \forall(u, v) \in \widetilde{H}(\mathcal{Q})^{2} .
$$

On the boundary $\partial \mathcal{Q}$ in the Orz plane, we denote by $n=\left(n_{r}, n_{z}\right)^{t}$ the unit out normal vector and by $\tau=\left(-n_{z}, n_{r}\right)^{t}$ the tangential vector. The tangential gradient operator on $\partial \mathcal{Q}$ is defined by $\nabla_{\tau}:=$ $\nabla-n \partial_{n}=\tau(\tau \cdot \nabla)$. Then we have in particular $\nabla u \cdot \nabla v=\partial_{n} u \partial_{n} v+\nabla_{\tau} u \cdot \nabla_{\tau} v$ on $\partial \mathcal{Q}$

Lemma 3.4. Assume that $\mu$ and $\sigma$ are constant in $\mathcal{Q}$, that $u(\mathcal{Q}) \in \widetilde{H}(\mathcal{Q})$ satisfies in the weak sense

$$
-\operatorname{div}\left(\frac{1}{\mu r} \nabla u\right)-\frac{i \omega \sigma}{r} u=0 \quad \text { in } \mathcal{Q},
$$

that $v(\mathcal{Q}) \in \widetilde{H}(\mathcal{Q})$, and that their material derivatives $\left(u^{\prime}(\theta), v^{\prime}(\theta)\right)$ and shape derivatives $(U(\theta), V(\theta))$ exist. Suppose in addition that the Hessians $D^{2} u$ and $D^{2} v$ are in $L^{2}(\mathcal{Q})^{2 \times 2}$. Then the shape derivative of $\alpha(\mathcal{Q})(u(\mathcal{Q}), v(\mathcal{Q}))$ denoted by $\beta(\theta)$ exists for all admissible perturbations $\theta$ and is given by

$$
\begin{aligned}
\beta(\theta)= & \alpha(\mathcal{Q})\left(u^{\prime}(\theta), v(\mathcal{Q})\right)+\alpha(\mathcal{Q})(u(\mathcal{Q}), V(\theta)) \\
& +\int_{\partial \mathcal{Q}}\left\{(\theta \cdot n)\left(\frac{1}{\mu r} \nabla_{\tau} u \cdot \nabla_{\tau} \bar{v}-\frac{\mathrm{i} \omega \sigma}{r} u \bar{v}\right)-\left(\frac{1}{\mu r} \partial_{n} u\left(\theta \cdot \nabla_{\tau} \bar{v}\right)\right)\right\} \mathrm{d} s .
\end{aligned}
$$

The proof of this lemma makes use of the shape derivative techniques which are extensively presented in [1,10]. Readers may refer to [15, Chapter 2] for technical details.

Assumption 3.5. Under the same assumptions as in Proposition 3.3 for $\mu, \sigma, J$ and $\theta$, we assume in addition that $(\mu, \sigma)$ are piecewise constant and equal to constants $\left(\mu_{i}, \sigma_{i}\right)$ on each subdomain $\Omega_{i}(i \in \Lambda)$ of $\Omega$, and that the boundaries $\partial \Omega_{i}(i \in\{d, v\})$ have $C^{1,1}$-regularity.

Proposition 3.6. Under Assumption 3.5, the material derivative $W(\theta)$ of $w$ satisfies

$$
\begin{aligned}
& a(W(\theta), \phi)=\int_{\Gamma}(\theta \cdot n)\left(\left[\frac{1}{\mu}\right] \frac{1}{r} \nabla_{\tau} w \cdot \nabla_{\tau} \bar{\phi}-\frac{\mathrm{i} \omega[\sigma]}{r} w \bar{\phi}\right) \mathrm{d} s \\
& \quad+\int_{\Omega_{d} \cup \Omega_{d}^{\mathrm{C}}}\left(\frac{1}{\mu r} \nabla(\theta \cdot \nabla w) \cdot \nabla \bar{\phi}-\frac{\mathrm{i} \omega \sigma}{r}(\theta \cdot \nabla w) \bar{\phi}\right) \mathrm{d} r \mathrm{~d} z \quad \forall \phi \in \widetilde{H}(\Omega) .
\end{aligned}
$$

Remark 3.7. The notation $\int_{\Omega_{d} \cup \Omega_{v}}$ means the integrals are evaluated separately on $\Omega_{d}$ and on $\Omega_{d}^{\complement}$. This is because $(\theta \cdot \nabla w)$ is not in the function space $\widetilde{H}(\Omega)$. In fact, the jump of $\mu$ through the interface $\Gamma$ yields the transmission condition $\left[\mu^{-1} \partial_{n} w\right]=0$ on $\Gamma$. Thus $(\theta \cdot \nabla w)$ is discontinuous on $\Gamma$

$$
[(\theta \cdot \nabla w)]=\left[(\theta \cdot n) \partial_{n} w+\left(\theta \cdot \nabla_{\tau} w\right)\right]=(\theta \cdot n)\left[\partial_{n} w\right]=(\theta \cdot n)[\mu]\left(\mu^{-1} \partial_{n} w\right) .
$$

However, from Remark 2.2, we have $\left.(\theta \cdot \nabla w)\right|_{\Omega_{i}} \in \widetilde{H}\left(\Omega_{i}\right)$ for $i \in\{s, t, d, v\}$. In consequence, the gradients in the right-hand-side of (15) are well-defined and the right-hand-side of (15) defines a bounded antilinear form on $\widetilde{H}(\Omega)$.

The assumption of $C^{1,1}$-regularity of $\partial \Omega_{i}(i \in\{d, v\})$ does not meet the real setting of the ECT problem, since the deposit is attached to the shell side of the tube (see Figure 3). However, singularities that could occur at the points where $\Gamma$ touches the outer tube wall $\left\{r=r_{t_{2}}\right\}$ do not show up in numerical tests in Section 4.4. Actually, the regularization method introduced in Section 4.2 reduces the singular effect of these problematic zones all the less. 
Proof. We write the sesquilinear form $a(\cdot, \cdot)$ in (5) as the sum of forms on subdomains where $\mu$ and $\sigma$ are constant

$$
a(w, \varphi)=\sum_{i \in \Lambda} \alpha_{i}\left(\Omega_{i}\right)(w, \varphi)
$$

where $\alpha_{i}(\mathcal{Q})(\cdot, \cdot)$ is defined as $\alpha(\mathcal{Q})(\cdot, \cdot)$ in (12) with $\mu=\mu_{i}$ and $\sigma=\sigma_{i}$. We will also denote by $\beta_{i}(\theta)$ the shape derivative associated with $\alpha_{i}$. We choose the test function $\varphi$ on $\Omega_{\theta}$ such that $\phi=\varphi \circ(\operatorname{Id}+\theta)$ on $\Omega$. Thus, the material derivative of $\varphi$ vanishes. Note that the support of $\theta$ is contained in $\Omega_{d} \cup \Omega_{v}$, that the relation $w^{\prime}(\theta)=W(\theta)-\theta \cdot \nabla w$ holds on $\Omega_{d}$ and $\Omega_{d}^{\complement}$ respectively, and that the solution $w$ satisfies the transmission conditions $[w]=\left[\mu^{-1} \partial_{n} w\right]=0$ on $\Gamma$. Under the assumptions on $\mu, \sigma$ and $\Gamma$, we have in particular that the Hessian $D^{2} w$ is in $L^{2}\left(\Omega_{i}\right)^{2 \times 2}$ for $i \in\{d, v\}$ due to [17, Theorem 4.20] (or see Remark 2.2). By using a density argument, we can assume the test function $\left.\varphi\right|_{\Omega_{i}} \in \mathcal{C}^{\infty}\left(\Omega_{i}\right)$ without losing the generality, such that $D^{2} \phi \in L^{2}\left(\Omega_{i}\right)^{2 \times 2}(i \in\{d, v\})$. Then we apply Lemma (3.4) to each subdomain and get shape derivative of $a(w, \varphi)$

$$
\begin{aligned}
\sum_{i \in \Lambda} \beta_{i}(\theta)= & \sum_{i \in \Lambda} \alpha_{i}\left(\Omega_{i}\right)\left(w^{\prime}(\theta), \phi\right)-\int_{\Gamma}\left[(\theta \cdot n)\left(\frac{1}{\mu r} \nabla_{\tau} w \cdot \nabla_{\tau} \bar{\phi}-\frac{\mathrm{i} \omega \sigma}{r} w \bar{\phi}\right)-\left(\frac{1}{\mu r} \partial_{n} w\left(\theta \cdot \nabla_{\tau} \bar{\phi}\right)\right)\right] \mathrm{d} s \\
= & a(W(\theta), \phi)-\int_{\Omega_{d} \cup \Omega_{d}^{\complement}}\left(\frac{1}{\mu r} \nabla(\theta \cdot \nabla w) \cdot \nabla \bar{\phi}-\frac{\mathrm{i} \omega \sigma}{r}(\theta \cdot \nabla w) \bar{\phi}\right) \mathrm{d} r \mathrm{~d} z \\
& -\int_{\Gamma}(\theta \cdot n)\left(\left[\frac{1}{\mu}\right] \frac{1}{r} \nabla_{\tau} w \cdot \nabla_{\tau} \bar{\phi}-\frac{\mathrm{i} \omega[\sigma]}{r} w \bar{\phi}\right) \mathrm{d} s .
\end{aligned}
$$

On the other hand, since the support of the source $J$ is contained in $\Omega_{s}$, the shape derivative of the right-hand-side of the variational formulation (5) vanishes. Hence, we get the result (15).

\subsection{Shape derivative of the impedance}

Now that we have the shape and material derivatives of the solution, we can compute the shape derivative of the measured impedances. Let $w$ be the solution of problem (5) with coefficients $(\mu, \sigma)$ and $w^{0}$ the solution in a deposit free-case, i.e. with coefficients $(\mu, \sigma)=\left(\mu^{0}, \sigma^{0}\right)$. We shall denote by $\alpha_{0}(\mathcal{Q})$ the sesquilinear form $\alpha(\mathcal{Q})$ for $(\mu, \sigma)=\left(\mu^{0}, \sigma^{0}\right)$. Following (4) we define the impedance measurement as

$$
\triangle Z(\Omega)=\frac{2 \pi}{\mathrm{i} \omega I^{2}} \int_{\Omega_{d}}\left(\left(\frac{1}{\mu}-\frac{1}{\mu^{0}}\right) \frac{\nabla w \cdot \nabla w^{0}}{r}-\mathrm{i} \omega\left(\sigma-\sigma^{0}\right) \frac{w w^{0}}{r}\right) \mathrm{d} r \mathrm{~d} z .
$$

Proposition 3.8. Under Assumption 3.5, the shape derivative of $\triangle Z(\Omega)$ is well defined and is given by

$$
\begin{aligned}
\triangle Z^{\prime}(\theta)= & \frac{2 \pi}{\mathrm{i} \omega I^{2}} \int_{\Omega_{d}}\left(\left(\frac{1}{\mu}-\frac{1}{\mu^{0}}\right) \frac{1}{r} \nabla(W(\theta)-\theta \cdot \nabla w) \cdot \nabla w^{0}-\frac{\mathrm{i} \omega\left(\sigma-\sigma^{0}\right)}{r}(W(\theta)-\theta \cdot \nabla w) w^{0}\right) \mathrm{d} r \mathrm{~d} z \\
& +\frac{2 \pi}{\mathrm{i} \omega I^{2}} \int_{\Gamma}(\theta \cdot n)\left(\left(\frac{1}{\mu}-\frac{1}{\mu^{0}}\right) \frac{1}{r} \nabla w \cdot \nabla w^{0}-\frac{\mathrm{i} \omega\left(\sigma-\sigma^{0}\right)}{r} w w^{0}\right) \mathrm{d} s .
\end{aligned}
$$

where $w^{\prime}(\theta)$ and $W(\theta)$ are respectively the shape and material derivative of $w$.

Proof. Since $\mu, \sigma, \mu^{0}$ and $\sigma^{0}$ are constant in $\Omega_{d}$, from (4) and the definition of $\alpha$ in (12) we have

$$
\frac{\mathrm{i} \omega I^{2}}{2 \pi} \triangle Z=\alpha\left(\Omega_{d}\right)\left(w, \overline{w^{0}}\right)-\alpha\left(\Omega_{d}\right)\left(w^{0}, \bar{w}\right)
$$

The field $w^{0}$ for the deposit-free case is invariant under the shape deformation (Id $\left.+\theta\right)$ (since $\mu^{0}$ and $\sigma^{0}$ are invariant under the shape deformation $(\mathrm{Id}+\theta)$ ). Thus its shape derivative is zero and consequently its material derivative is $W^{0}(\theta)=\theta \cdot \nabla w^{0}$ due to the relation (8). In $\Omega_{d}$ the field $w$ satisfies equation (13) with material parameters $\mu, \sigma$, while $w^{0}$ satisfies (13) with $\mu^{0}$ and $\sigma^{0}$. We note that $w, w^{0} \in H_{l o c}^{2}\left(\Omega_{d}\right)$ due to Remark 2.2. Applying Lemma 3.4 and after some computations (c.f. [15] for details) one gets

$$
\frac{\mathrm{i} \omega I^{2}}{2 \pi} \triangle Z^{\prime}(\theta)=\alpha\left(\Omega_{d}\right)\left(w^{\prime}, \overline{w^{0}}\right)-\alpha_{0}\left(\Omega_{d}\right)\left(w^{\prime}, \overline{w^{0}}\right)+\int_{\Gamma}(\theta \cdot n)\left(\left(\frac{1}{\mu}-\frac{1}{\mu^{0}}\right) \frac{\nabla w \cdot \nabla w^{0}}{r}-\frac{\mathrm{i} \omega\left(\sigma-\sigma^{0}\right)}{r} w w^{0}\right) \mathrm{d} s .
$$

This is exactly expression (18) considering (8). 


\subsection{Expression of the shape derivative using the adjoint state}

The expression of the gradient $\triangle Z^{\prime}(\theta)$ shown in (18) contains not only a boundary integral on $\Gamma$ whose integrand depends explicitly on the shape perturbation $\theta$, but also a volume integral on $\Omega_{d}$ with the shape or material derivative of $w$ in the integrand which depends implicitly on $\theta$ via the variational problem (15). We shall consider here the Hadamard representation of cost functional derivatives using an appropriately defined adjoint state which allows to have an expression of $\triangle Z^{\prime}(\theta)$ as a boundary integral on $\Gamma$ with integrand explicitly dependent on $\theta$. This expression is much more appropriate for the numerical scheme that we shall use for the inverse problem.

We define the sesquilinear form

$$
a^{*}(u, v):=\overline{a(v, u)} \quad \forall(u, v) \in \widetilde{H}(\Omega)^{2} .
$$

and we introduce the adjoint problem associated with $w^{0}$ as finding $p \in \widetilde{H}(\Omega)$ such that

$$
a^{*}(p, q)=\int_{\Omega_{d}}\left(\left(\frac{1}{\mu}-\frac{1}{\mu^{0}}\right) \frac{1}{r} \nabla \overline{w^{0}} \cdot \nabla \bar{q}+\frac{\mathrm{i} \omega\left(\sigma-\sigma^{0}\right)}{r} \overline{w^{0}} \bar{q}\right) \mathrm{d} r \mathrm{~d} z \quad \forall q \in \widetilde{H}(\Omega) .
$$

In particular, $p$ satisfies the jump conditions

$$
[p]=0 \quad \text { and } \quad\left[\mu^{-1} \partial_{n} p\right]=-\left(\frac{1}{\mu}-\frac{1}{\mu^{0}}\right) \partial_{n} \overline{w^{0}} \quad \text { on } \Gamma .
$$

Problem (20) has the same structure as (5) since its right-hand-side defines a bounded anti-linear form on $\widetilde{H}(\Omega)$. Therefore one can conclude:

Proposition 3.9. Let $w^{0} \in \widetilde{H}(\Omega)$ be the solution to the eddy current problem (5) in a deposit-free case, i.e. with $\left(\mu^{0}, \sigma^{0}\right)$ instead of $(\mu, \sigma)$. Then, under the same assumptions as in Corollary [2.1] for $\mu$ and $\sigma$, the variational formulation (20) has a unique solution $p$ in $\widetilde{H}(\Omega)$.

Proposition 3.10. Under Assumption [3.5, if $p$ is the solution to the adjoint problem (20), then the shape derivative of the impedance $\triangle Z$ given by (4) writes

$$
\begin{aligned}
\triangle Z^{\prime}(\theta)=\frac{2 \pi}{\mathrm{i} \omega I^{2}} & \int_{\Gamma} \frac{(\theta \cdot n)}{r}\left\{\left[\frac{1}{\mu}\right] \nabla_{\tau} w \cdot \nabla_{\tau}\left(\bar{p}-w^{0}\right)\right. \\
& \left.-[\mu]\left(\mu^{-1} \partial_{n} w\right)\left(\left(\mu^{0}\right)^{-1}\left(\partial_{n} \bar{p}\right)_{+}-\left(\mu^{0}\right)^{-1} \partial_{n} w^{0}\right)-\mathrm{i} \omega[\sigma] w\left(\bar{p}-w^{0}\right)\right\} \mathrm{d} s,
\end{aligned}
$$

where $w$ (resp. $w^{0}$ ) is the solution to the weighted eddy current problem (5) with (resp. without) deposits.

Remark 3.11. With the same argument presented in Remark 2.2, one observes that the adjoint state $p$ has also $H^{2}$-regularity in $\Omega_{d}$ and $\Omega_{v}$ since $w^{0} \in H^{2}\left(\Omega_{d}\right)$. In particular, the tangential and normal traces of $\left.\nabla p\right|_{\Omega_{i}}(i \in\{d, v\})$ on $\Gamma$ are well-defined and have $H^{1 / 2}$-regularity. Therefore the boundary integration in formula (22) is well-defined.

Proof. It is sufficient on one hand to evaluate (20) with test function $q=W(\theta) \in \widetilde{H}(\Omega)$ or $q=(\theta \cdot \nabla w)$ with the jump condition (16), and on the other hand to set $\phi=p$ in the formulaton(15) and consider the jump condition for $p$ (21).

\section{Shape reconstruction of deposits using a gradient method}

\subsection{Cost functional}

We denote by $Z$ the impedance measurement either in absolute mode $\left(Z_{F A}\right)$ or in differential mode $\left(Z_{F 3}\right)$. Giving the ECT signals $Z_{\text {meas }}(\zeta)$ for $\zeta \in\left[z_{\min }, z_{\max }\right]$, the inverse problem aims to approximate the real deposit domain by an estimate $\Omega_{d}$ in simulation so that the ETC signals $Z\left(\Omega_{d}, \zeta\right)$ reproduced with $\Omega_{d}$ approach $Z_{\text {meas }}(\zeta)$. This naturally motivates us to define a least square cost functional

$$
\mathcal{J}\left(\Omega_{d}\right)=\int_{z_{\min }}^{z_{\max }}\left|Z\left(\Omega_{d} ; \zeta\right)-Z_{\text {meas }}(\zeta)\right|^{2} \mathrm{~d} \zeta
$$


and apply shape optimization using gradient descent. One computes its shape derivative

$$
\mathcal{J}^{\prime}(\theta)=\int_{z_{\min }}^{z_{\max }} 2 \Re\left(Z^{\prime}(\theta) \overline{\left(Z\left(\Omega_{d} ; \zeta\right)-Z_{\text {meas }}(\zeta)\right)}\right) \mathrm{d} \zeta,
$$

where $Z^{\prime}(\theta)$ (either $Z_{F A}^{\prime}(\theta)$ or $Z_{F 3}^{\prime}(\theta)$ ) is a linear combination of $\triangle Z_{k l}^{\prime}$. According to (22)

$$
\begin{aligned}
\triangle Z_{k l}^{\prime}(\theta)=\frac{2 \pi}{\mathrm{i} \omega I^{2}} & \int_{\Gamma} \frac{(\theta \cdot n)}{r}\left\{\left[\frac{1}{\mu}\right] \nabla_{\tau} w_{k} \cdot \nabla_{\tau}\left(\overline{p_{l}}-w_{l}^{0}\right)\right. \\
& \left.\quad-[\mu]\left(\mu^{-1} \partial_{n} w_{k}\right)\left(\left(\mu^{0}\right)^{-1}\left(\partial_{n} \overline{p_{l}}\right)_{+}-\left(\mu^{0}\right)^{-1} \partial_{n} w_{l}^{0}\right)-\mathrm{i} \omega[\sigma] w_{k}\left(\overline{p_{l}}-w_{l}^{0}\right)\right\} \mathrm{d} s .
\end{aligned}
$$

The shape derivative of the cost functional $\mathcal{J}$ can be written as

$$
\mathcal{J}^{\prime}\left(\Omega_{d}\right)(\theta)=\frac{2 \pi}{\omega I^{2}} \int_{\Gamma}(n \cdot \theta) g \mathrm{~d} s,
$$

where $g=g_{F A}=g_{11}+g_{21}$ or $g=g_{F 3}=g_{11}-g_{22}$ according to the measuring mode with

$$
\begin{aligned}
g_{k l} & =\int_{z_{\min }}^{z_{\max }} \Re\left\{\overline { ( Z ( \Omega _ { d } ; \zeta ) - Z _ { m e s } ( \zeta ) ) } \frac { 1 } { r } \left(\left[\frac{1}{\mu}\right] \nabla_{\tau} w_{k} \cdot \nabla_{\tau}\left(\overline{p_{l}}-w_{l}^{0}\right)\right.\right. \\
& \left.\left.-[\mu]\left(\mu^{-1} \partial_{n} w_{k}\right)\left(\frac{1}{\mu^{0}}\left(\partial_{n} \overline{p_{l}}\right)_{+}-\frac{1}{\mu^{0}} \partial_{n} w_{l}^{0}\right)-\mathrm{i} \omega[\sigma] w_{k}\left(\overline{p_{l}}-w_{l}^{0}\right)\right)\left.\right|_{\zeta}\right\} \mathrm{d} \zeta .
\end{aligned}
$$

We remark in particular that if one choose $\theta$ such that

$$
\theta=-\gamma g n \quad \text { on } \Gamma,
$$

where $\gamma$ is a positive constant, then $\theta$ is a minimizing direction of $\mathcal{J}$ for $\gamma$ sufficiently small.

\subsection{Regularization of the descent direction}

For an arbitrary parametrization of $\Omega_{d}$, a regularization of the descent direction is in general needed since the shape increment given by (24) may cause a singularity on $\Gamma$ (see the numerical experiments below). We propose to use the $H^{1}(\Gamma)$ boundary regularization by solving the following problem for $\lambda \in H^{1}(\Gamma)^{2}$ :

$$
\lambda-\alpha \triangle_{\Gamma} \lambda=g n \quad \text { on } \Gamma,
$$

where $\triangle_{\Gamma}$ is the boundary Laplace-Beltrami operator applied element-wise to $\lambda$ and $\alpha>0$ is a regularization parameter. The equivalent variational formulation of (25) is,

$$
\forall \psi \in H^{1}(\Gamma)^{2} \quad \int_{\Gamma}\left(\lambda \cdot \psi+\alpha \nabla_{\tau} \lambda \cdot \nabla_{\tau} \psi\right) \mathrm{d} s=\int_{\Gamma} g n \cdot \psi \mathrm{d} s .
$$

Therefore, $\lambda$ is two orders more regular than $g n$. If we take $\theta$ such that

$$
\theta=-\gamma \lambda \quad \text { on } \Gamma
$$

one verifies that it is also a descent direction

$$
\mathcal{J}^{\prime}\left(\Omega_{d}\right)(\theta)=-\gamma \frac{2 \pi}{\omega I^{2}} \int_{\Gamma}\left(|\lambda|^{2}+\alpha\left|\nabla_{\tau} \lambda\right|^{2}\right) \mathrm{d} s \leq 0 .
$$

\subsection{Inversion algorithm}

The inversion procedure is done as follows:

- Initialize with a deposit domain $\Omega_{d}^{0}$. Choose $\delta>0$ as a threshold in the stopping rule according to the noise level of the data, and $\epsilon>0$ as an upper bound for the size of the descent gradient. 
- Step $k$ :

1. Solve the direct problems (5) for the different positions $\zeta$ of the coils using the deposit shape $\Omega_{d}^{k}$ and test the stopping rule

$$
\mathcal{J}\left(\Omega_{d}^{k}\right) \leq \delta^{2} \int_{z_{\min }}^{z_{\max }}\left|Z_{\text {meas }}(\zeta)\right|^{2} \mathrm{~d} \zeta
$$

2. Solve the adjoint problems (20) for the different coil positions and for the deposit shape $\Omega_{d}^{k}$ then evaluate the corresponding $g$.

3. Get a regularized descent direction $\theta^{k}$ (see (25) and (27)). The parameter $\gamma$ in (24) is evaluated at the first step $(k=1)$ such that $\gamma \max |g| \leq \epsilon$.

4. Go to step $k+1$ with a deposit domain

$$
\Omega_{d}^{k+1}=\left(\mathrm{Id}+\theta^{k}\right) \Omega_{d}^{k}
$$

\subsection{Numerical tests}

We shall consider here some numerical inversion tests for deposits for geometrical configurations depicted in Figure2, The numerical values of physical parameters are chosen according to the materials (e.g. tube and magnetite) and the setting used for non-destructive eddy current testing of steam generators:

- The tube is defined by $\Omega_{t}=\left\{(r, z): r_{t_{1}} \leq r \leq r_{t_{2}}\right\}$ with $r_{t_{1}}=9.84 \mathrm{~mm}, r_{t_{2}}=11.11 \mathrm{~mm}$. Its conductivity is $\sigma_{t}=9.7 \times 10^{5} \mathrm{~S} / \mathrm{m}$ and its magnetic permeability is $\mu_{t}=1.01 \mu_{v}$, where $\mu_{v}$ is the permeability of vacuum.

- The deposit has in general a relatively low conductivity: $\sigma_{d}=1 \times 10^{4} \mathrm{~S} / \mathrm{m}$. It can be magnetic: permeability $\mu_{d}=10 \mu_{v}$ or non-magnetic: $\mu_{d}=\mu_{v}$.

- The operating frequency for the coils is $\omega=100 \mathrm{kHz}$, the dimensions of one coil are $0.67 \mathrm{~mm}$ in length (radial direction) and $2 \mathrm{~mm}$ in height (axial direction). Both the two coils are located $7.83 \mathrm{~mm}$ away from the $z$-axis and there is a distance of $0.5 \mathrm{~mm}$ between them.

We remark that the above-described test setting (low frequency and high conductivity regime) allows to apply eddy current approximation to the full model (see for example [2]).

The numerical forward problem is set on a bounded domain $B_{r_{*}, z_{*}}$ with $r_{*}=30 \mathrm{~mm}$ and $z_{*}=41 \mathrm{~mm}$. It is solved using FreeFem ++ [14] with P1 finite elements and an adapted mesh (using the command adaptmesh). The mesh is adapted according to the solution at each step of the iteration such that the relative interpolation error is less than $1 \%$. The number of degrees of freedom is around 1000 (see Figure 4(a). To avoid committing an inverse crime when generating synthetic data for the inversion process, we use a refined mesh to generate the impedance measurements as given observation data (see Figure 4(b)]. The number of degrees of freedom of P1 finite element on this mesh is about 6000 . Validation of the numerical forward model can be found in [13.

For the inversion we use impedance measurements either in the pseudo-absolute mode

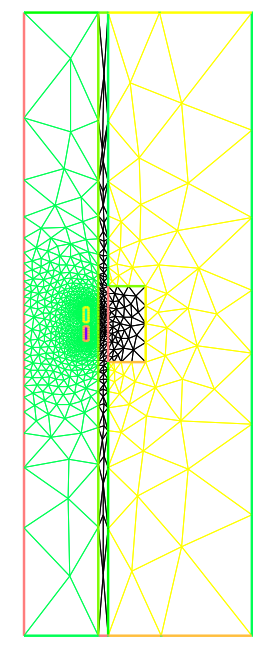

(a)

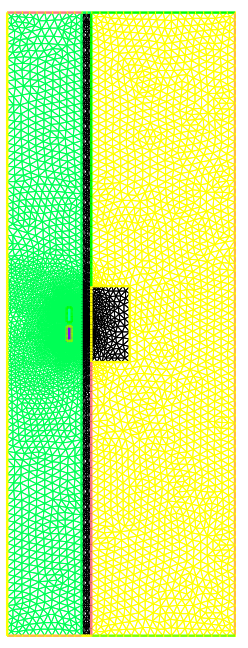

(b)
Figure 4: Examples of meshes used for inversion and generation of data. (a) Adapted mesh for solving the forward problem in the inversion process. (b) Refined mesh for generating the observation data. 
(FA) or in the differential mode (F3). The number of vertical measurement points involved in the reconstruction will be specified for each experiment. The vertical measurement positions chosen for the inversion are localized around the vertical center of the target deposit. This is justified by the fact that in practice, one can immediately determine the vertical location of the deposit from observed signal variations while performing the vertical scan of the tube. The algorithm parameters for the stopping rule is set to $\delta=1 \%, 2 \%$ or $3 \%$ in different cases and the increment magnitude is set to $\epsilon=5 \times 10^{-4}$.

Finally let us note that in all subsequent figures, the target deposit shape is shown in green while the reconstructed shape using the inverse algorithm is in red.

\subsubsection{Parametrized shape reconstruction}

We consider a non-magnetic deposit. We assume that the deposit is rectangular in the semi-plan $\mathbb{R}_{+}^{2}$. Then its shape can be parametrized by its thickness in the $r$-direction and the positions in the $z$-direction of its two horizontal sides. The target shape has $5 \mathrm{~mm}$ in thickness, and its horizontal sides are at $\pm 5 \mathrm{~mm}$.

To reconstruct both the thickness and the two vertical positions of the horizontal sides of the rectangular deposit, we use either FA or F3 signals at 41 probe positions with a distance of $1 \mathrm{~mm}$ between two neighboring positions. Figure 5 and Table 1 show the results. We initialize the inverse algorithm with either a small guess (Figure $5(\mathrm{a})$ or a large one (Figure $5(\mathrm{~d})$ ). The result from the small guess using FA signal after 71 iterations is shown in Figure 5(b), and that using F3 signal after 43 iterations is shown in Figure 5(c). From a large guess, we get the reconstruction result in Figure $5(\mathrm{e})$ using FA signal after 24 iterations, and that in Figure $5(\mathrm{e})$ using F3 signal after 112 iterations. In Figures 5(g) $5(\mathrm{j})$ we observe the decrease of the relative error of signals during iterations. However, the decrease of the shape relative error (the difference of the characteristic functions of the target deposit domain and the reconstructed domain measured in the $L^{2}$ norm) may stagnate around $10 \%$, which means that the information from the impedance measurements is no longer sufficient to distinguish the reconstructed shape from the target shape.

\begin{tabular}{lccc}
\hline & thickness & vertical position 1 & vertical position 2 \\
\hline target shape & $5 \mathrm{~mm}$ & $5 \mathrm{~mm}$ & $-5 \mathrm{~mm}$ \\
from small guess, FA & $5.236 \mathrm{~mm}$ & $4.872 \mathrm{~mm}$ & $-4.870 \mathrm{~mm}$ \\
from small guess, F3 & $4.882 \mathrm{~mm}$ & $5.017 \mathrm{~mm}$ & $-5.017 \mathrm{~mm}$ \\
from large guess, FA & $5.015 \mathrm{~mm}$ & $5.041 \mathrm{~mm}$ & $-5.039 \mathrm{~mm}$ \\
from large guess, F3 & $5.123 \mathrm{~mm}$ & $4.983 \mathrm{~mm}$ & $-4.982 \mathrm{~mm}$ \\
\hline
\end{tabular}

Table 1: Parameter reconstructions of a rectangular non-magnetic deposit.

\subsubsection{Reconstruction of deposits with arbitrary shapes}

In this section we consider the reconstruction of the deposit without a priori knowledge on its shape.

In Figure 6 the target non-magnetic deposit shape is a rectangle. Since we do not have any information of the shape, we take a small semi-disc as the initial guess in the inversion algorithm. We use either FA or F3 signals for inversion at 41 probe positions with a distance of $1 \mathrm{~mm}$ between each two neighboring positions. The algorithm without boundary regularization using FA signal is blocked due to singularities on the interface between the deposit and the vacuum (Figure 6(b)) .

To regularize the gradient using the method in Section 4.2, we take $\alpha=1 \times 10^{-5}$ as the regularization parameter in the boundary regularization problem (25). This is an ad hoc choice. Our numerical tests suggest that relatively moderate variations of this parameter does not affect the final result. The regularized algorithm using FA signals ends after 201 iterations with a good estimate (Figure 6(c)) and that using F3 signals gives the result shown in Figure 6(d) after 412 iterations. We also show in Figures 6(e) and 6(f) the decrease of the cost functional, the absolute value of gradient and the relative error on the shape during iterations.

In Figure 7 we show the reconstructions of a non-magnetic semi-disc issued from different initial shapes (Figures $7(\mathrm{a})$ or $7(\mathrm{c})$ using FA signals. The corresponding reconstruction results shown in Figure 


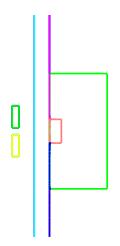

(a)

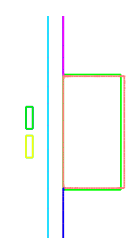

(b)

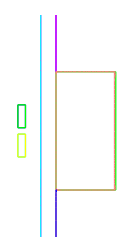

(c)

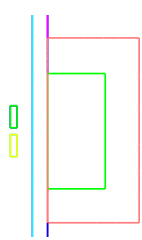

(d)

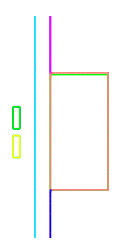

(e)

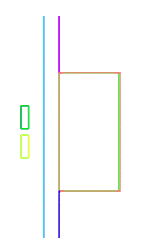

(f)

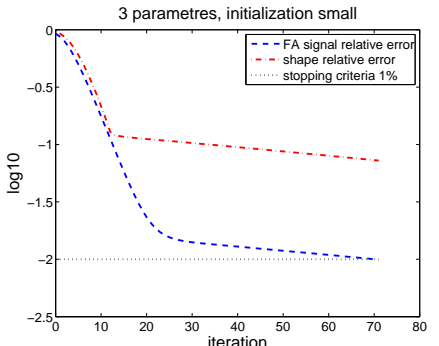

(g) FA

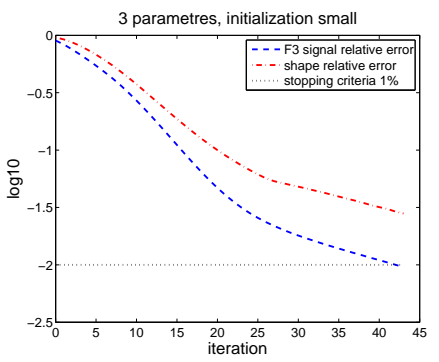

(i) F3

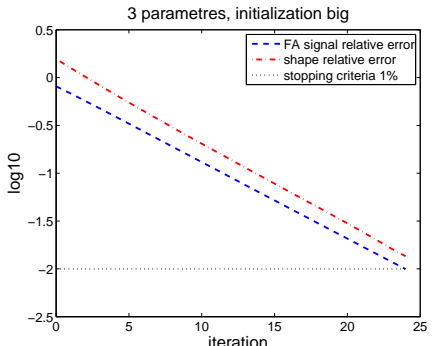

(h) FA

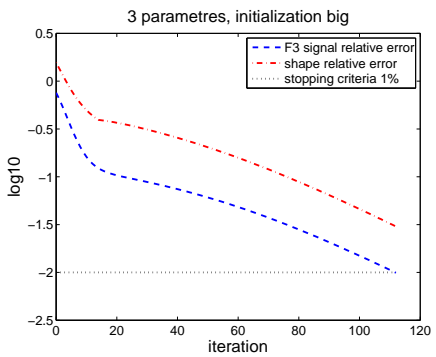

(j) F3

Figure 5: Parameter reconstructions of a rectangular non-magnetic deposit. $\delta=1 \%$. (a) Initialization with a small guess. (b) Reconstruction from small initial guess using FA signals. (c) Reconstruction from small initial guess using F3 signals. (d) Initialization with a large guess. (e) Reconstruction from large initial guess using FA signals. (f) Reconstruction from large initial guess using F3 signals. (g)-to-(j) Relative errors on signal and shape during iterations.

7(b) (37 iterations) and in Figure 7(d) (52 iterations) for the non-magnetic deposits are satisfying, as we can observe the decrease of the relative errors of signals and deposit shapes in Figures $7(\mathrm{e})$ and $7(\mathrm{f})$,

Finally Figure 8 shows the reconstruction of a non convex deposit shape using differential mode (F3) impedance signals. For the non-magnetic deposit (Figures $8(\mathrm{a})-8(\mathrm{~b})$ ), we choose the stopping threshold $\delta=2 \%$ and the algorithm ends after 139 iterations. For the magnetic deposit (Figures $8(\mathrm{c})-8(\mathrm{~d}))$, with $\delta=3 \%$, the algorithm ends after 786 iterations.

\subsubsection{Stability to noisy data}

In this section we test the robustness of the above shape reconstruction method with regard to the given data noise (FA or F3 signals). Supposing that the relative artificial noise level equals $\eta$, we choose for the inversion algorithm a stopping rule such that the relative signal error is below $\eta+\delta$ where $\delta$ is the stopping rule for the case without data noise that we used in the previous tests.

Figure 9 and Table 2 show parameter reconstructions of a non-magnetic rectangular deposit after artificially adding a random noise vector to the simulated signal data. We recall that the stopping rule is $\delta=1 \%$ for the case without artificial noise. So here we choose the stopping rules such that the relative signal error is inferior to the artificial noise level plus $\delta=1 \%$. We observe that when the artificial noise level is under $5 \%$, the results are quite satisfying even compared to the reconstruction results from data without artificial noise. (Figure 5 and Table 1).

Figure 10 shows the shape reconstruction results of a general non-magnetic deposit from artificially 


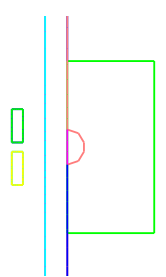

(a)

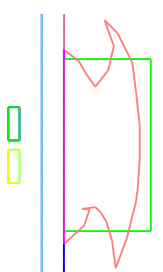

(b)

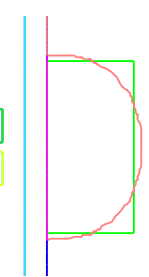

(c)

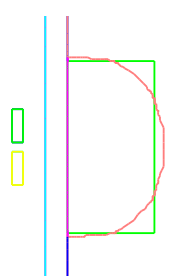

(d)

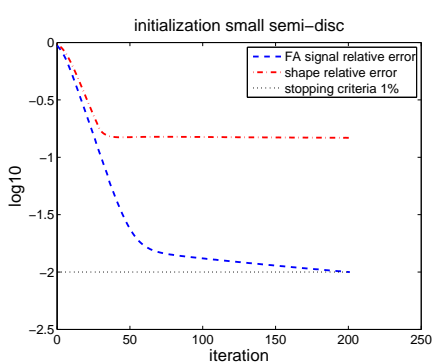

(e) FA

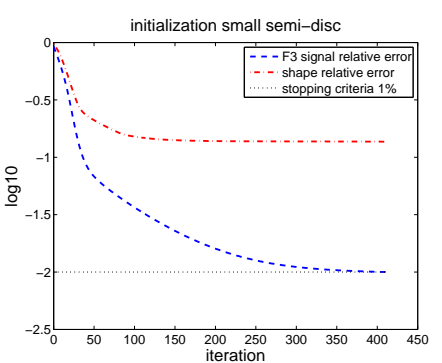

(f) F3

Figure 6: Reconstruct a rectangular non-magnetic deposit. $\delta=1 \%$. (a) Initialization with a small semi-disc. (b) Blocked non-regularized inversion algorithm. (c) Regularized reconstruction using FA signals. (d) Regularized reconstruction using F3 signals. (e) - (f) Signal and shape relative errors during regularized reconstruction iterations.

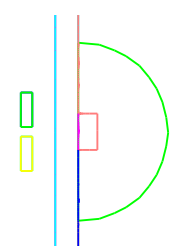

(a)

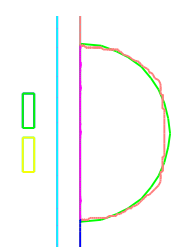

(b)

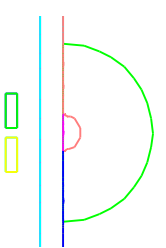

(c)

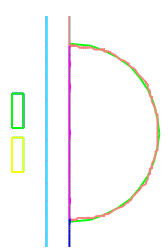

(d)

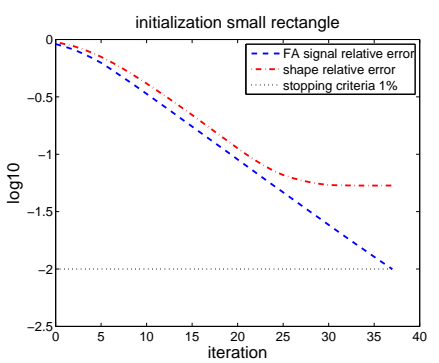

(e) initial: small rectangle

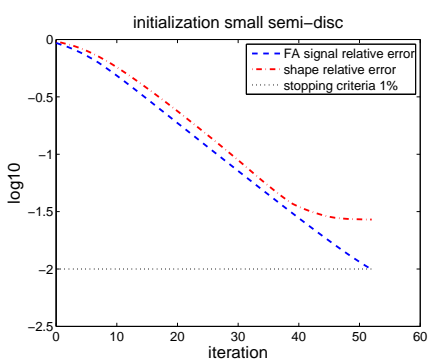

(f) initial: small semi-disc

Figure 7: Reconstruct a non-magnetic semi-disc shaped deposit. $\delta=1 \%$. (a) Initialization with a small rectangle. (b) Reconstruction from initial small rectangle. (c) Initialization with a small semi-disc. (d) Reconstruction from initial small semi-disc. (e) - (f) Relative signal and shape errors during iterations.

noised F3 signals. The stopping rule of the inversion algorithm for the case without artificial noise was $\delta=2 \%$. So here the algorithm is stopped once the relative error of F3 signals is below the artificial noise level plus $\delta=2 \%$. The reconstruction results are also satisfying.

\section{On the reconstruction of deposit conductivity and permeability}

The conductivity and the permeability are the two critical physical parameters which characterize the material nature of the deposit. The exact values of these parameters, crucial for the modeling, the 


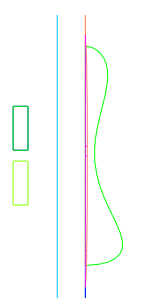

(a)

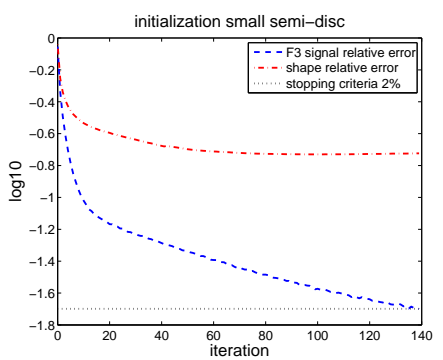

(e) non-magnetic (b)

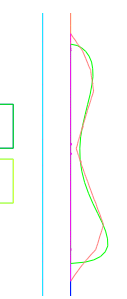

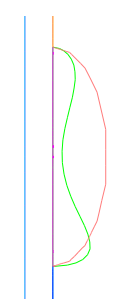

(c)

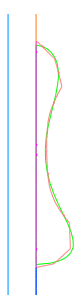

(d)

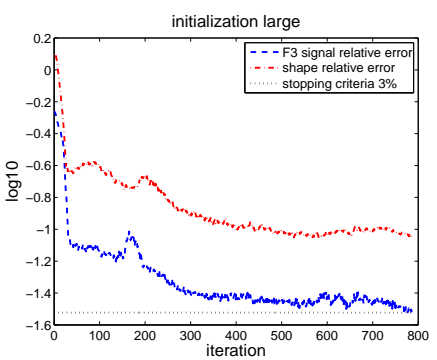

(f) magnetic

Figure 8: Reconstruct a deposit with a non-convex shape. $\delta=2 \%$ for the non-magnetic case, $\delta=3 \%$ for the magnetic case. (a) Initialization of a non-magnetic deposit with a thin line. (b) Reconstruction of the non-magnetic deposit. (c) Initialization of a magnetic deposit with a large shape. (d) Reconstruction of the magnetic deposit. (e) - (f) Relative signal and shape errors during iterations.

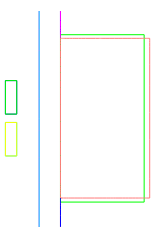

(a) $1 \%$ noise

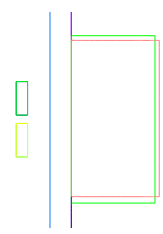

(b) $5 \%$ noise

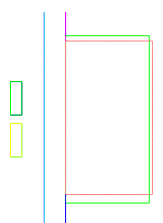

(c) $10 \%$ noise

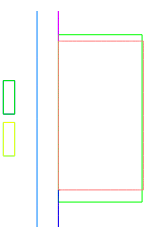

(d) $20 \%$ noise

Figure 9: Parameter reconstructions of a rectangular non-magnetic deposit from artificially noised FA signals.

\begin{tabular}{lccc}
\hline noise level & thickness & vertical position 1 & vertical position 2 \\
\hline target shape & $5 \mathrm{~mm}$ & $5 \mathrm{~mm}$ & $-5 \mathrm{~mm}$ \\
$1 \%$ & $5.336 \mathrm{~mm}$ & $4.788 \mathrm{~mm}$ & $-4.766 \mathrm{~mm}$ \\
$5 \%$ & $5.286 \mathrm{~mm}$ & $4.746 \mathrm{~mm}$ & $-4.645 \mathrm{~mm}$ \\
$10 \%$ & $5.232 \mathrm{~mm}$ & $4.719 \mathrm{~mm}$ & $-4.527 \mathrm{~mm}$ \\
$20 \%$ & $5.138 \mathrm{~mm}$ & $4.682 \mathrm{~mm}$ & $-4.325 \mathrm{~mm}$ \\
\hline
\end{tabular}

Table 2: Parameter reconstructions of a rectangular non-magnetic deposit from artificially noised FA signals.

simulation and the reconstruction of the deposit is usually not known with a high precision in the industrial context. In this section we discuss the reconstruction of these parameters for known shapes. The simultaneous reconstruction of the parameters and the shape is discussed in the last section. 


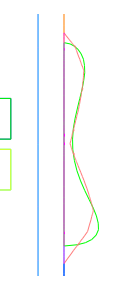

(a) $1 \%$ noise

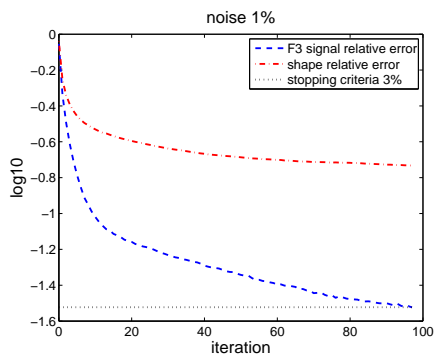

(d) $1 \%$ noise (b) $5 \%$ noise

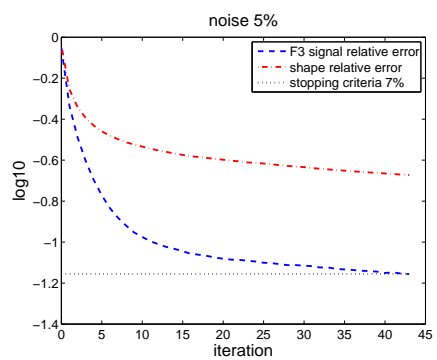

(e) $5 \%$ noise (c) $10 \%$ noise

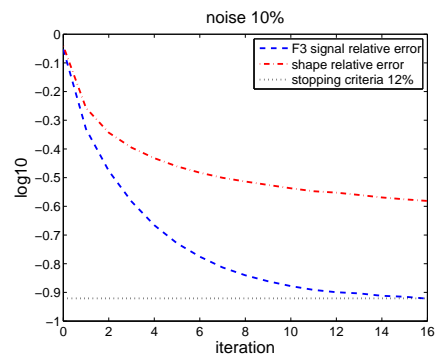

(f) $10 \%$ noise

Figure 10: Reconstruct a deposit with a non-convex shape from artificially noised F3 signals. (a) -to(c) Reconstructed shapes from signals of different artificial noise level. (d) -ro- (f) Relative signal and shape errors during iterations.

\subsection{The cost functional derivative with respect to the conductivity}

We consider the eddy current problem (5). We denote by $\delta w$ the variation of $w$ due to a small increment of the conductivity $\sigma_{d} \rightarrow \sigma_{d}+\delta \sigma_{d}$ that is assumed to be constant. Therefore,

$$
\int_{\Omega}\left(\frac{1}{\mu r} \nabla(w+\delta w) \cdot \nabla \bar{\varphi}-\frac{\mathrm{i} \omega\left(\sigma+\delta \sigma_{d} \chi_{\Omega_{d}}\right)}{r}(w+\delta w) \bar{\varphi}\right) \mathrm{d} r \mathrm{~d} z=\int_{\Omega} \mathrm{i} \omega J \bar{\varphi} \mathrm{d} r \mathrm{~d} z \quad \forall \varphi \in \widetilde{H}(\Omega)
$$

where $\chi_{\Omega_{d}}$ is the index function of the domain $\Omega_{d}$. After developing this formulation, the terms of order zero of the variation give the original problem (5). The derivative of $w$ with respect to $\sigma_{d}$ writes:

$$
\partial_{\sigma} w:=\lim _{\delta \sigma_{d} \rightarrow 0} \delta w / \delta \sigma_{d}
$$

where the limit holds in $\widetilde{H}(\Omega)$. Then the terms of first order of the variation in the above formulation as $\delta \sigma_{d}$ goes to zero imply

$$
\int_{\Omega}\left(\frac{1}{\mu r} \nabla\left(\partial_{\sigma} w\right) \cdot \nabla \bar{\varphi}-\frac{\mathrm{i} \omega \sigma}{r}\left(\partial_{\sigma} w\right) \bar{\varphi}\right) \mathrm{d} r \mathrm{~d} z=\int_{\Omega_{d}} \frac{\mathrm{i} \omega}{r} w \bar{\varphi} \mathrm{d} r \mathrm{~d} z .
$$

Now we consider the impedance measurement given by (4). If $\partial_{\sigma}\left(\triangle Z_{k l}\right)$ is its derivative with respect to $\sigma_{d}$, then

$$
\partial_{\sigma}\left(\triangle Z_{k l}\right)=\frac{2 \pi}{\mathrm{i} \omega I^{2}} \int_{\Omega_{d}}\left(\left(\frac{1}{\mu}-\frac{1}{\mu^{0}}\right) \frac{\nabla\left(\partial_{\sigma} w_{k}\right) \cdot \nabla w^{0}}{r}-\mathrm{i} \omega\left(\sigma-\sigma^{0}\right) \frac{\left(\partial_{\sigma} w_{k}\right) w^{0}}{r}-\mathrm{i} \omega \frac{w_{k} w^{0}}{r}\right) \mathrm{d} r \mathrm{~d} z .
$$

Similarly, we denote by $\partial_{\sigma} \mathcal{J}$ the derivative of the cost functional $\mathcal{J}$ given by (23) with respect to the variation of $\sigma_{d}$. We get

$$
\partial_{\sigma} \mathcal{J}=\int_{z_{\min }}^{z_{\max }} 2 \Re\left\{\partial_{\sigma} Z\left(\Omega_{d} ; \zeta\right)\left(\overline{Z\left(\Omega_{d} ; \zeta\right)-Z_{\text {meas }}(\zeta)}\right)\right\} \mathrm{d} \zeta,
$$

where according to the impedance measuring mode,

$$
\partial_{\sigma} Z\left(\Omega_{d} ; \zeta\right)=\left\{\begin{array}{l}
\partial_{\sigma} Z_{F A}=\frac{\mathrm{i}}{2}\left(\partial_{\sigma}\left(\triangle Z_{11}\right)+\partial_{\sigma}\left(\triangle Z_{21}\right)\right), \\
\partial_{\sigma} Z_{F 3}=\frac{\mathrm{i}}{2}\left(\partial_{\sigma}\left(\triangle Z_{11}\right)-\partial_{\sigma}\left(\triangle Z_{22}\right)\right) .
\end{array}\right.
$$


To minimize the cost functional with respect to $\sigma_{d}$ we shall use a descent gradient method based of a numerical evaluation of the derivative provided by (31).

\subsection{Derivative with respect to the magnetic permeability}

Similarly to the previous section, we consider here a small increment of the deposit magnetic permeability $\mu_{d} \rightarrow \mu_{d}+\delta \mu_{d}$ which leads to a small variation of the field $w \rightarrow \delta w$. Then from (5) we derive

$$
\int_{\Omega}\left(\frac{1}{\left(\mu+\delta \mu_{d} \chi_{\Omega_{d}}\right) r} \nabla(w+\delta w) \cdot \nabla \bar{\varphi}-\frac{\mathrm{i} \omega \sigma}{r}(w+\delta w) \bar{\varphi}\right) \mathrm{d} r \mathrm{~d} z=\int_{\Omega} \mathrm{i} \omega J \bar{\varphi} \mathrm{d} r \mathrm{~d} z .
$$

If we denote by

$$
\partial_{\mu} w:=\lim _{\delta \mu_{d} \rightarrow 0} \delta w / \delta \mu_{d}
$$

where the limit is understood with respect to the $\widetilde{H}(\Omega)$ norm, then one verifies that $\partial_{\mu} w$ satisfies

$$
\int_{\Omega}\left(\frac{1}{\mu r} \nabla\left(\partial_{\mu} w\right) \cdot \nabla \bar{\varphi}-\frac{\mathrm{i} \omega \sigma}{r}\left(\partial_{\mu} w\right) \bar{\varphi}\right) \mathrm{d} r \mathrm{~d} z=\int_{\Omega_{d}} \frac{1}{\mu^{2} r} \nabla w \cdot \nabla \bar{\varphi} \mathrm{d} r \mathrm{~d} z \quad \forall \varphi \in \widetilde{H}(\Omega) .
$$

Then the derivative of the impedance measurement $\triangle Z_{k l}$ with regard to the deposit magnetic permeability, is given by the following expression:

$$
\partial_{\mu}\left(\triangle Z_{k l}\right)=\frac{2 \pi}{\mathrm{i} \omega I^{2}} \int_{\Omega_{d}}\left(\left(\frac{1}{\mu}-\frac{1}{\mu^{0}}\right) \frac{\nabla\left(\partial_{\mu} w_{k}\right) \cdot \nabla w_{l}^{0}}{r}-\mathrm{i} \omega\left(\sigma-\sigma^{0}\right) \frac{\left(\partial_{\mu} w_{k}\right) w_{l}^{0}}{r}-\frac{\nabla w_{k} \cdot \nabla w_{l}^{0}}{\mu^{2} r}\right) \mathrm{d} r \mathrm{~d} z .
$$

If $\partial_{\mu} \mathcal{J}$ represents the derivative of the cost functional $\mathcal{J}$ with respect to the variation of $\mu_{d}$, then

$$
\partial_{\mu} \mathcal{J}=\int_{z_{\min }}^{z_{\max }} 2 \Re\left\{\partial_{\mu} Z\left(\Omega_{d} ; \zeta\right)\left(\overline{Z\left(\Omega_{d} ; \zeta\right)-Z_{\text {meas }}(\zeta)}\right)\right\} \mathrm{d} \zeta,
$$

due to (23), where according to the impedance measurement mode,

$$
\partial_{\mu} Z\left(\Omega_{d} ; \zeta\right)=\left\{\begin{array}{l}
\partial_{\mu} Z_{F A}=\frac{\mathrm{i}}{2}\left(\partial_{\mu}\left(\triangle Z_{11}\right)+\partial_{\mu}\left(\triangle Z_{21}\right)\right) \\
\partial_{\mu} Z_{F 3}=\frac{\mathrm{i}}{2}\left(\partial_{\mu}\left(\triangle Z_{11}\right)-\partial_{\mu}\left(\triangle Z_{22}\right)\right)
\end{array}\right.
$$

To minimize the cost functional with respect to $\mu_{d}$ we shall also use a descent gradient method based of a numerical evaluation of the derivative provided by (34).

\subsection{Numerical tests}

\subsubsection{Reconstruction of the conductivity or of the magnetic permeability}

We first consider the reconstruction of the conductivity of a non-magnetic deposit $\left(\mu_{d}=\mu_{v}\right)$ with $\sigma_{d}=1 \times 10^{4} \mathrm{~S} / \mathrm{m}$ in a known shape (a $5 \mathrm{~mm} \times 10 \mathrm{~mm}$ rectangle). We initialize the inversion algorithm with either a small guess of the conductivity $\left(5 \times 10^{3} \mathrm{~S} / \mathrm{m}\right)$ or a large guess $\left(3 \times 10^{4} \mathrm{~S} / \mathrm{m}\right)$. The reconstruction results using FA signals at one probe position are given in Figures 11(a) - 11(b).

We then want to reconstruct the magnetic permeability of a magnetic deposit with $\sigma_{d}=1 \times 10^{4} \mathrm{~S} / \mathrm{m}$, $\mu_{d}=10 \mu_{v}$ and in a known shape (a $2 \mathrm{~mm} \times 10 \mathrm{~mm}$ rectangle) at the shell side of the tube. We initialize the inversion algorithm with either a small guess of the magnetic permeability $\left(2 \mu_{v}\right)$ or a large guess $\left(15 \mu_{v}\right)$. Results are given in Figure $11(\mathrm{c})-11(\mathrm{~d})$.

One observes that the reconstruction results for conductivity are satisfying (relative error of conductivity is less than $1 \%$ when the cost functional is under $10^{-4}$, i.e. when relative difference of FA signals between given data and simulation is less than 1\%), while the proposed methods are not satisfactory for permeability reconstruction. In fact, these results show that eddy current signals are more sensitive to conductivity changes than to permeability changes. 


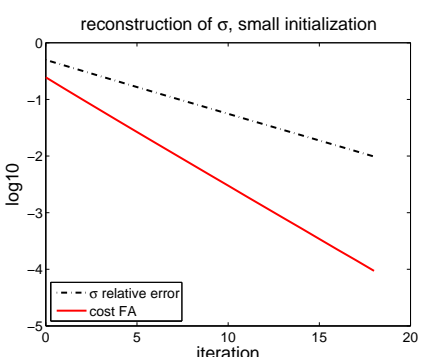

(a)

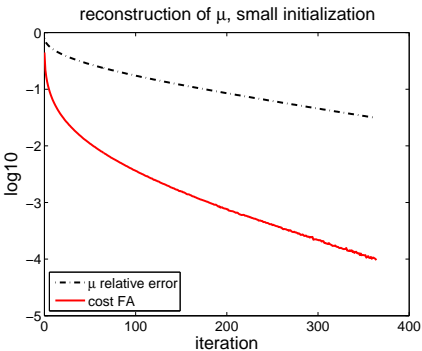

(c)

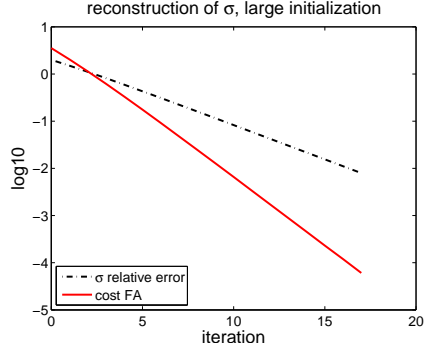

(b)

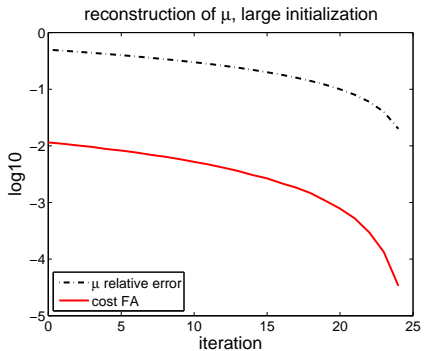

(d)

Figure 11: Reconstruction of the conductivity or of the magnetic permeability using FA signals. (a) - (b) Conductivity reconstruction for a non-magnetic deposit with fixed $\mu_{d}=\mu_{v}$. (a) Initial guess $\sigma_{d}^{\text {guess }}=5 \times 10^{3} \mathrm{~S} / \mathrm{m}$, result $\sigma_{d}=9901 \mathrm{~S} / \mathrm{m}$ after 18 iterations. (b) Initial guess $\sigma_{d}^{\text {guess }}=3 \times 10^{4} \mathrm{~S} / \mathrm{m}$, result $\sigma_{d}=10079 \mathrm{~S} / \mathrm{m}$ after 17 iterations. (c) - (d) Permeability reconstruction for a magnetic deposit with fixed $\sigma_{d}=10^{4} \mathrm{~S} / \mathrm{m}$. (c) Initial guess $\mu_{d}^{\text {guess }}=2 \mu_{v}$, result $\mu_{d}=9.69 \mu_{v}$ after 364 iterations. (d) Initial guess $\mu_{d}^{\text {guess }}=15 \mu_{v}$, result $\mu_{d}=10.2 \mu_{v}$ after 24 iterations.

\subsubsection{Simultaneous reconstruction of conductivity and the magnetic permeability}

We try to reconstruct here both the conductivity and the magnetic permeability with FA signals at one probe position. The conductivity and the magnetic permeability of the target rectangular deposit $(2 \mathrm{~mm} \times 10 \mathrm{~mm})$ are respectively $\sigma_{t}=1 \times 10^{4} \mathrm{~S} / \mathrm{m}, \mu_{t}=10 \mu_{v}$. The initialization of these two parameters can be either small or large. The results are shown in Figure 12 and Table 3.

\begin{tabular}{lccc}
\hline & initial guess & reconstructed & number of iterations \\
\hline target deposit & & $(10000,10)$ & \\
test 1 & $(5000,5)$ & $(9309,9.65)$ & 44 \\
test 2 & $(5000,20)$ & $(10666,10.37)$ & 12 \\
test 3 & $(20000,5)$ & $(10649,9.78)$ & 42 \\
test 4 & $(20000,20)$ & $(10921,10.24)$ & 13 \\
\hline
\end{tabular}

Table 3: Reconstruction of the conductivity and the relative magnetic permeability $\left(\sigma_{d}(S / m), \mu_{d}\right)$ using FA signals.

We observe that the simultaneous reconstruction results are not accurate even if the normalized cost functional is under $10^{-4}$. This is explained by the extremely low dependence of the cost functional with repect to simultaneous variations of the two parameters. This is clearly indicated by Figure $13(\mathrm{a})$. We hence conclude that the these eddy-current measurements are not really suited to determine physical parameters. 


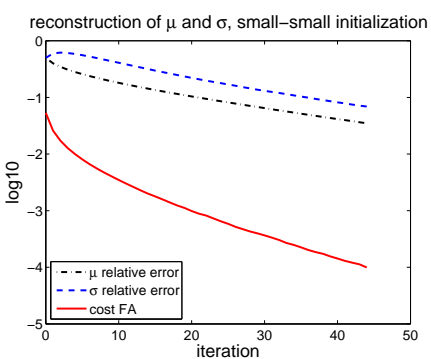

(a) test 1

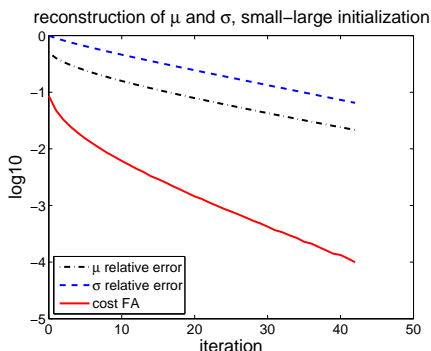

(c) test 3

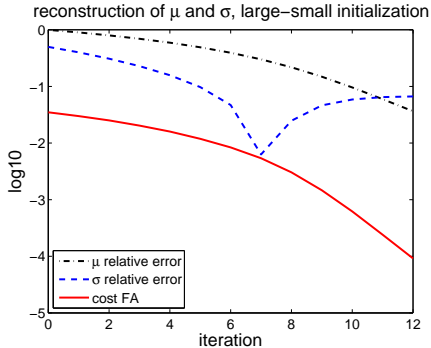

(b) test 2

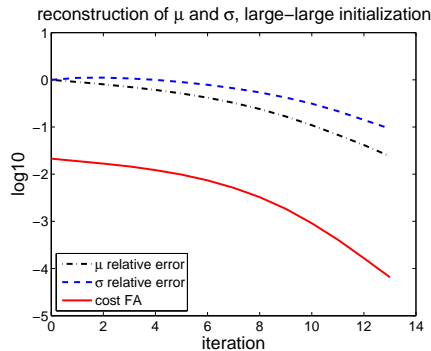

(d) test 4

Figure 12: Reconstruction of both the conductivity and the magnetic permeability using FA signals.

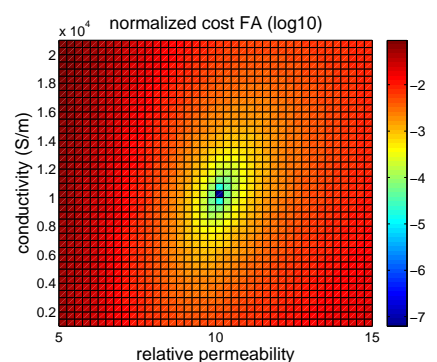

(a) conductivity and permeability

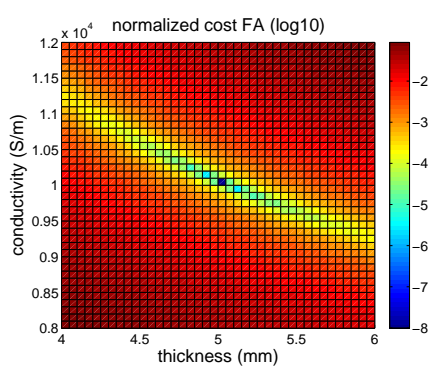

(b) conductivity and thickness

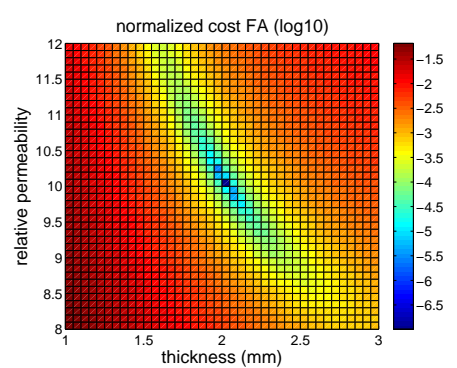

(c) permeability and thickness

Figure 13: Interference between physical parameters and shape parameter. (a) Between conductivity and magnetic permeability. (b) Between conductivity and thickness. (c) Between permeability and thickness.

\section{On the reconstruction of the shape and physical parameters}

We would like to discuss here the possibility of reconstructing simultaneously the conductivity (or the magnetic permeability) and the shape of the deposit by coupling the inversion algorithm for shape reconstruction in Section 4.3 and that for conductivity (magnetic permeability) reconstruction. We consider the simplest cases in which the deposit shape is a rectangle with unknown thickness but with fixed horizontal sides.

In the first case with unknown conductivity and thickness, the target deposit is a $5 \mathrm{~mm} \times 10 \mathrm{~mm}$ rectangle with the $\sigma_{d}=10^{4} \mathrm{~S} / \mathrm{m}$ and $\mu_{d}=\mu_{v}$. For rectangular deposits with the range of thickness from $4 \mathrm{~mm}$ to $6 \mathrm{~mm}$ and the range of conductivity from $8 \times 10^{3} \mathrm{~S} / \mathrm{m}$ to $1.2 \times 10^{4} \mathrm{~S} / \mathrm{m}$, we show in Figure 13(b) the value (in $\log _{10}$ ) of the cost functional of the absolute mode impedance measurements (FA) normalized with regard to the FA impedance measurement of the target deposit.

In the second case where the magnetic permeability and the thickness are to reconstruct, the target deposit is a $2 \mathrm{~mm} \times 10 \mathrm{~mm}$ rectangle with $\sigma_{d}=10^{4} \mathrm{~S} / \mathrm{m}$ and $\mu_{d}=10 \mu_{v}$. For rectangular deposits with the range of thickness from $1 \mathrm{~mm}$ to $3 \mathrm{~mm}$ and the range of relative magnetic permeability from 8 to 12 , we show similarly the normalized cost functional for FA signals in Figure 13(c),

In both two cases the interferences between the physical parameters and the geometrical parameter (the thickness) are too important to hope obtaining a precise reconstruction. For instance, $\sigma=0.95 \times$ 
$10^{4} \mathrm{~S} / \mathrm{m}$ and a thickness $=5.6 \mathrm{~mm}$ would lead to a relative magnitude of the cost functional of order $10^{-4}$ which reaches the stopping threshold of the inversion algorithm. Similarly, $\mu=0.95 \mu_{v}$ and a thickness $=2.2 \mathrm{~mm}$ would lead to a relative magnitude of the cost functional of order $10^{-4}$.

\begin{tabular}{lrrrr}
\hline & $\sigma(S / \mathrm{m})$ & $\mu / \mu_{v}$ & initial guess & reconstruction \\
\hline target deposit & $1 \times 10^{4}$ & 10 & & $2 \mathrm{~mm}$ \\
test 1 & $0.98 \times 10^{4}$ & 10 & $0.5 \mathrm{~mm}$ & $1.91 \mathrm{~mm}$ \\
test 2 & $0.98 \times 10^{4}$ & 10 & $4 \mathrm{~mm}$ & $2.08 \mathrm{~mm}$ \\
test 3 & $1 \times 10^{4}$ & 9.8 & $0.5 \mathrm{~mm}$ & $1.96 \mathrm{~mm}$ \\
test 4 & $1 \times 10^{4}$ & 9.8 & $4 \mathrm{~mm}$ & $2.13 \mathrm{~mm}$ \\
\hline
\end{tabular}

Table 4: Reconstruction of thickness of a rectangular deposit with wrong values of the conductivity or the magnetic permeability using FA signals.

However, with a good initial guess of the conductivity and the permeability, shape reconstruction of deposits yields reasonable results. We observe in Table 4 that a small error in $\sigma$ or in $\mu(2 \%)$ would still lead to accurate reconstruction of rectangular deposit shape. In Figure 14, we show the reconstruction results of general shapes for non-magnetic deposits (magnetic permeability equals to $\mu_{v}$ ) with a good guess of the conductivity - either $\sigma_{1}=0.98 \times 10^{4} \mathrm{~S} / \mathrm{m}$ or $\sigma_{2}=1.02 \times 10^{4} \mathrm{~S} / \mathrm{m}$ against the exact value of the conductivity which is $10^{4} \mathrm{~S} / \mathrm{m}$. With the threshold in the stopping rule $\delta=10^{-4}$ and an initialization with small semi-disc (see Figure 7(c) for the reconstruction of a semi-disc (Figures 14(a), 14(b) and 14(e)) or $\delta=2 \%$ and an initialization with a thin line (see Figure $8(\mathrm{a})$ for the reconstruction of a curved shape (Figures 14(c), 14(d) and 14(f), we observe that the reconstructed shapes are good approximations of the target shapes.

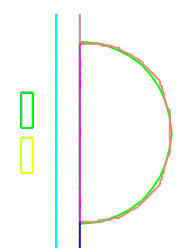

(a)

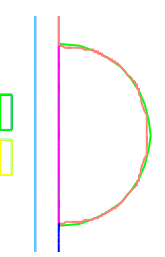

(b)

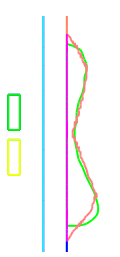

(c)

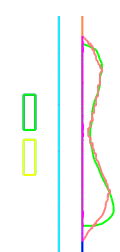

(d)

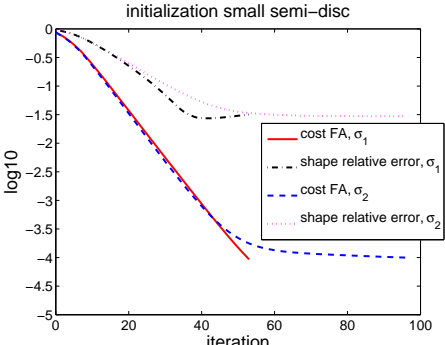

(e) semi-disc

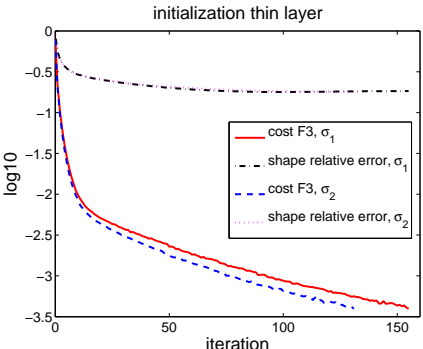

(f) curved shape

Figure 14: Reconstruction with inexact but good guess of conductivity $\left(\sigma_{1}=0.98 \times 10^{4} \mathrm{~S} / \mathrm{m}\right.$ or $\sigma_{2}=$ $1.02 \times 10^{4} \mathrm{~S} / \mathrm{m}$ ) against exact value $\sigma_{d}=10^{4} \mathrm{~S} / \mathrm{m}$ for non-magnetic deposits. (a) Reconstruction of a semi-disc using FA signals with conductivity guess $\sigma_{1}$ after 53 iterations. (b) Reconstruction of a semidisc using FA signals with conductivity guess $\sigma_{2}$ after 96 iterations. (c) Reconstruction of a curved shape using F3 signals with conductivity guess $\sigma_{1}$ after 155 iterations. (d) Reconstruction of a curved shape using F3 signals with conductivity guess $\sigma_{2}$ after 133 iterations. (e) - (f) Relative signal and shape errors during iterations. 


\section{References}

[1] G. Allaire. Conception optimale de structures, volume 58 of Mathématiques $\& 3$ Applications. Springer, Berlin, 2007. With the collaboration of Marc Schoenauer in the writing of Chapter 8.

[2] A. Alonso Rodríguez and A. Valli. Eddy current approximation of Maxwell equations, volume 4 of MSEAA. Modeling, Simulation and Applications. Springer-Verlag Italia, Milan, 2010. Theory, algorithms and applications.

[3] B. A. Auld, S. R. Jefferies, and J. C. Moulder. Eddy-current signal analysis and inversion for semielliptical surface cracks. Journal of Nondestructive Evaluation, 7(1-2):79-94, 1988.

[4] B. A. Auld and J. C. Moulder. Review of advances in quantitative eddy current nondestructive evaluation. Journal of Nondestructive Evaluation, 18:3-36, 1999. 10.1023/A:1021898520626.

[5] R. Beissner. Approximate model of eddy-current probe impedance for surface-breaking flaws. Journal of Nondestructive Evaluation, 7:25-34, 1988.

[6] J. Blitz. Electrical and Magnetic Methods of Non-Destructive Testing. Non-destructive evaluation series. Chapman \& Hall, 1997.

[7] M. Bonnet and B. B. Guzina. Sounding of finite solid bodies by way of topological derivative. International Journal for Numerical Methods in Engineering, 61(13):2344-2373, 2004.

[8] L. Chatellier, S. Dubost, F. Peisey, Y. Goussard, and R. Guichard. Characterization of small depth surface breaking defects with eddy current sensor measurements. In Proc. ECNDT 2006, 2006.

[9] N. Chaulet. Modèles d'impédance généralisée en diffraction inverse. PhD thesis, École Polytechnique, 2012.

[10] M. C. Delfour and J.-P. Zolésio. Shapes and Geometries, volume 4 of Advances in Design and Control. Society for Industrial and Applied Mathematics (SIAM), Philadelphia, PA, 2001. Analysis, differential calculus, and optimization.

[11] O. Dorn and D. Lesselier. Level set methods for inverse scattering-some recent developments. Inverse Problems, 25(12):125001, 11, 2009.

[12] B. B. Guzina and M. Bonnet. Topological derivative for the inverse scattering of elastic waves. The Quarterly Journal of Mechanics and Applied Mathematics, 57(2):161-179, 2004.

[13] H. Haddar, Z. Jiang, and A. Lechleiter. Artificial boundary conditions for axisymmetric eddy current probe problems. Computers 83 Mathematics with Applications, 68(12, Part A):1844 - 1870, 2014.

[14] F. Hecht. New development in Freefem++. Journal of Numerical Mathematics, 20(3-4):251-265, 2012.

[15] Z. Jiang. Some inversion methods applied to non-destructive testings of steam generator via eddy current probe. PhD thesis, École Polytechnique, 2014.

[16] J.-L. Lions and E. Magenes. Problèmes aux limites non homogènes et applications. Vol. 1. Travaux et Recherches Mathématiques, No. 17. Dunod, Paris, 1968.

[17] W. McLean. Strongly Elliptic Systems and Boundary Integral Equations. Cambridge University Press, Cambridge, 2000.

[18] F. Murat and J. Simon. Etude de problèmes d'optimal design. In J. Cea, editor, Optimization Techniques Modeling and Optimization in the Service of Man Part 2, volume 41 of Lecture Notes in Computer Science, pages 54-62. Springer Berlin Heidelberg, 1976.

[19] F. Murat and J. Simon. Sur le contrôle par un domaine géométrique. Technical Report LAN-76015, Université Pierre et Marie Curie (Paris), 1976. 
[20] D. Nicolas. Couplage de méthodes d'échantillonnage et de méthodes d'optimisation de formes pour des problèmes de diffraction inverse. PhD thesis, École Polytechnique, 2012.

[21] O. Pantz. Sensibilité de l'équation de la chaleur aux sauts de conductivité. Comptes Rendus Mathématique. Académie des Sciences. Paris, 341(5):333-337, 2005.

[22] G. Pichenot, D. Prémel, T. Sollier, and V. Maillot. Development of a 3d electromagnetic model for eddy current tubing inspection: Application to steam generator tubing. In AIP Conference Proceedings, volume 700, pages 321-328, 2003. 10.1063/1.1711640.

[23] F. Santosa. A level-set approach for inverse problems involving obstacles. European Series in Applied and Industrial Mathematics: Control, Optimation and Calculus of Variations, 1:17-33, 1995/96.

[24] A. Trillon. Reconstruction de défauts à partir de données issues de capteurs à courants de Foucault avec modèle direct différentiel. PhD thesis, École Centrale de Nantes, 2010.

[25] R. Zorgati, B. Duchêne, D. Lesselier, and F. Pons. Eddy current testing of anomalies in conductive materials. i. qualitative imaging via diffraction tomography techniques. IEEE Transaction on Magnetics, 27(6):4416-4437, 1991.

[26] R. Zorgati, D. Lesselier, B. Duchêne, and F. Pons. Eddy current testing of anomalies in conductive materials. ii. quantitative imaging via deterministic and stochastic inversion techniques. IEEE Transactions on Magnetics, 28(3):1850-1862, 1992. 